\title{
AN OUTLINE OF THE THEORY OF PSEUDOANALYTIC FUNCTIONS ${ }^{1}$
}

\section{LIPMAN BERS}

1. Introduction. Pseudoanalytic functions are, roughly speaking, solutions of generalized Cauchy-Riemann equations. Such functions were considered by Picard $[67 ; 68]$ and by Beltrami $[3 ; 4]$, but the first significant result was obtained by Carleman [28] in 1933, and a systematic theory was formulated only recently. No comprehensive account is available in print and the main results have been announced only episodically and, in most cases, under unnecessarily restrictive hypotheses. In this paper we shall outline the main features of this theory.

The theory of analytic functions of a complex variable occupies a central place in analysis and it is not surprising that mathematical literature abounds in generalizations. In some generalizations one extends the domain of the functions considered, or their range, or both (functions of several complex variables, analytic functions with values in a vector space or an algebra, analytic functions of hypercomplex variables, analytic operators, etc.). If we restrict ourselves to functions from plane domains to plane domains, or, more generally, from Riemann surfaces to Riemann surfaces, we encounter two well known and very useful generalizations of analytic functions: interior functions and quasi-conformal functions. Interior functions $[47 ; 60 ; 61 ; 62 ; 63 ; 78 ; 86]$ have all topological properties of analytic functions and no others. As a matter of fact, they may be defined as functions which can be made analytic by a homeomorphism of the domain of definition. Quasi-conformal functions $[2 ; 27 ; 46 ; 66 ; 75 ; 79]$ are interior functions subject to an additional metric condition. ${ }^{2}$ If the functions are assumed to be continuously differentiable mappings, this additional condition requires that infinitesimal circles be taken into infinitesimal ellipses of uniformly bounded eccentricity.

An address delivered before the Annual Meeting of the Society in Pittsburgh, Pennsylvania, on December 28, 1955, by invitation of the Committee to Select Hour Speakers for Annual and Summer Meetings; received by the editors January 30, 1956.

${ }^{1}$ Some of the results presented have been obtained during work under contract DA-30-069-ORD-835 with the Office of Ordnance Research, United States Army. Reproduction in whole or in part permitted for any purpose of the United States Government.

${ }^{2}$ Some authors, to the present writer's regret, use the term pseudoanalytic for quasi-conformal functions. 
The class of quasi-conformal functions contains all analytic functions as well as many others. For this very reason the theory of quasiconformal functions can not have the inner rigidity and harmony of classical function theory. For example, quasi-conformal functions do not have the unique continuation property which Riemann considered to be the most characteristic feature of analytic functions. Pseudoanalytic functions, on the other hand, do possess the unique continuation property, and each class of pseudoanalytic functions has almost as much structure as the class of analytic functions. In particular, the operations of complex differentiation and complex integration have meaningful counterparts in the theory of pseudoanalytic functions, and this theory generalizes not only the Cauchy-Riemann approach to function theory but also that of Weierstrass.

The theory of pseudoanalytic functions was developed from the point of view of partial differential equations, much of the motivation being provided by problems in mechanics of continua. But in this paper we present pseudoanalytic functions axiomatically, as a generalization of classical function theory.

We hope to show that this generalization is not empty (that is, that it preserves essential features of the original theory), not trivial (that is, not a direct consequence of the original theory) and not isolated (that is, that it has applications which are of independent interest).

Remark. Analogues of complex differentiation and integration for an elliptic partial differential equation were used by Beltrami $[3 ; 4]$ in the special case of axially symmetric potentials. In a systematic way these concepts, as well as formal powers and power series, have been introduced in 1943 by Gelbart and the author $[21 ; 22 ; 23]$ for equations of a special form (cf. \$13). Markuševitch [65] observed that this formalism can be extended to general equations. The author's original version of a general theory of pseudoanalytic functions $[11 ; 12]$ was influenced by the work of Položii $[69 ; 70 ; 71 ; 72]$ and made essential use of Carleman's unique continuation theorem [28]. This theory applied to equations with Hölder continuously differentiable coefficients. It contained, in particular, a description of zeros and singularities of solutions. In this direction similar and somewhat stronger results have been obtained by Vekua $[81 ; 82 ; 84]$, whose work contains also many other important results, by Hartman and Wintner $[39 ; 40 ; 41 ; 42]$ and, in connection with mapping problems, by Gergen and Dressel $[36 ; 37 ; 38]$. Two gaps in the theory were filled by Agmon and the author [1] and by Protter [73]. A more general theory, applicable to equations with merely Hölder continuous coeffi- 
cients was formulated in [18]. The proofs of some of the results described below are not yet published. A monograph on pseudoanalytic functions is in preparation.

\section{Definition AND local properties of PSEUDOANALYTIC FUNCTIONS}

2. Definition of pseudoanalytic functions. The class $A_{D}$ of functions $w(z)$ which are analytic in a fixed domain $D$ can be characterized by four properties. (1) $A_{D}$ is a real vector space. (2) If $w(z)$ belongs to $A_{D}$ and $w\left(z_{0}\right)=0$ for some $z_{0}$ in $D$, then the quotient

$$
w(z) /\left(z-z_{0}\right)
$$

is continuous at $z_{0}$. (3) The functions 1 and $i$ belong to $A_{D}$. (4) $A_{D}$ is maximal with respect to properties (1), (2), (3).

In fact, the first three properties imply that the existence of the complex derivative $w^{\prime}(z)$ at every point of $D$ is necessary in order that a function $w$ should belong to $A_{D}$, and property (4) implies that this condition is sufficient.

Pseudoanalytic functions are obtained by a modification of the preceding definition $[11 ; 12 ; 18]$.

Let $F(z), G(z)$ be two continuous functions defined in a domain $D_{0}$ and satisfying the inequality

$$
\operatorname{Im}\{\overline{F(z)} G(z)\}>0 .
$$

(We write functions of $(x, y)$ as functions of $z=x+i y$, without implying analyticity. The bar over a complex number denotes the complex conjugate.) Let $A_{D}(F, G)$ be the maximal class of functions (defined in a domain $D \subset D_{0}$ ) such that properties (1) and (2) hold, and the "generators" $F$ and $G$ belong to $A_{D}(F, G)$. If $w(z)$ belongs to $A_{D}(F, G)$ we call $w$ an $(F, G)$ pseudoanalytic function of the first kind. It is clear that pseudoanalyticity of $w$ in $D$ implies its pseudoanalyticity in every subdomain of $D$. Pseudoanalyticity at a point is defined as pseudoanalyticity in some neighborhood of the point.

It follows from (2.1) that every function $w(z)$ defined in a subdomain of $D$, whether or not it belongs to $A_{D}(F, G)$, admits the unique representation

$$
w(z)=\phi(z) F(z)+\psi(z) G(z)
$$

where the functions $\phi, \psi$ are real-valued. It is convenient to associate with the function $w$ the function

$$
\omega(z)=\phi(z)+i \psi(z) .
$$


The correspondence between $w$ and $\omega$ is one-to-one. We denote it by writing

$$
\omega=* w, \quad w=\omega^{*} \quad(\bmod F, G) .
$$

If $w$ is $(F, G)$ pseudoanalytic of the first kind, we call $\omega$ pseudoanalytic of the second kind. In this case $w$ and $\omega$ should be considered as two representations of the same mathematical entity.

In general, pseudoanalytic functions do not form a multiplicative semi-group. Neither is a pseudoanalytic function of a pseudoanalytic function in general pseudoanalytic. As a matter of fact, there exists no nontrivial generalization of the analyticity concept for complexvalued functions of a complex variable in which unique continuation is preserved and the property of closure under multiplication and substitution is not lost. On the other hand, the definition of pseudoanalyticity is conformally covariant. ${ }^{3}$ If $\zeta=f(z), z=g(\zeta)$ is a conformal mapping of the domain $D$ onto $\Delta$, then a function $w(z)$ is $(F, G)$ pseudoanalytic if and only if the function $w[g(\zeta)]$ is $(\widetilde{F}, \widetilde{G})$ pseudoanalytic, where $\tilde{F}(\zeta), \tilde{G}(\zeta)$ are the transplanted generators: $\widetilde{F}=F[g(\zeta)], \widetilde{G}=G[g(\zeta)]$. This fact leads to a natural definition of pseudoanalyticity on a Riemann surface. In particular, $w(z)$ is $(F, G)$ pseudoanalytic at $z=\infty$ if and only if $\tilde{w}(\zeta)=w(1 / \zeta)$ is $(F(1 / \zeta)$, $G(1 / \zeta))$ pseudoanalytic at the origin.

Two pairs of generators, $(F, G)$ and $(\widehat{F}, \widehat{G})$, will be called equivalent if $\widehat{F}$ and $\widehat{G}$ are linear combinations, with real constant coefficients, of $F$ and $G$. The two pairs will be called equipotent if $\widehat{F}$ and $\widehat{G}$ are $(F, G)$ pseudoanalytic of the first kind. Equivalent generating pairs are always equipotent, and two equipotent pairs determine the same class of pseudoanalytic functions of the first kind. On the other hand, if $(F, G)$ is replaced by an equivalent (equipotent) pair, the functions of the second class undergo a fixed affine transformation with real constant (variable) coefficients. If there exists a function $H(z)$ such that $\widehat{F}=H F, \widehat{G}=H G,(F, G)$ and $(\widehat{F}, \widehat{G})$ are called similar. In this case the $(F, G)$ pseudoanalytic functions of the first kind are transformed into $(\widehat{F}, \widehat{G})$ pseudoanalytic functions by multiplication by the fixed function $H$; the class of pseudoanalytic functions of the second kind, however, is insensitive to a replacement of the generating pair by a similar one.

3. Differentiation and integration. $[10 ; 11 ; 18]$. The $(F, G)$ derivative of a function (2.2) is defined by the relation

${ }^{8}$ This shows that the use of complex numbers is here natural. Cf. however the comments of Kriszten [48]. 


$$
\begin{aligned}
\dot{w}(z) & =\frac{d_{(F, G)} w(z)}{d z} \\
& =\lim _{h \rightarrow 0} \frac{[\phi(z+h)-\phi(z)] F(z+h)+[\psi(z+h)-\psi(z)] G(z+h)}{h}
\end{aligned}
$$

provided that the limit to the right exists and is finite. It is easy to see that a function $w(z)$ is $(F, G)$ pseudoanalytic of the first kind in a domain $D$ if and only if it possesses an $(F, G)$ derivative at every point of $D$. The generators, which play the role of constants, have, of course, identically vanishing $(F, G)$ derivatives. It is clear that $(F, G)$ differentiability implies continuity. By letting $h$ in (3.1) approach zero first through real and then through purely imaginary values, one obtains the existence of the partial derivatives of $\phi$ and $\psi$, and the relations

$$
\begin{aligned}
& \phi_{z} F+\psi_{z} G=0, \\
& \phi_{z} F+\psi_{z} G=\dot{w}
\end{aligned}
$$

where the formal differential operators $\partial / \partial z, \partial / \partial \bar{z}$ are defined by the relations

$$
\frac{\partial}{\partial z}=\frac{1}{2}\left(\frac{\partial}{\partial x}-i \frac{\partial}{\partial y}\right), \quad \frac{\partial}{\partial \bar{z}}=\frac{1}{2}\left(\frac{\partial}{\partial x}+i \frac{\partial}{\partial y}\right) .
$$

Those familiar with the theory of elliptic partial differential equations will not be surprised to learn that continuity of the generators is not sufficient to insure the continuity of the $(F, G)$ derivatives. We shall therefore assume from now on that the generators $F$ and $G$ are Hölder continuous (that is, that they satisfy a uniform Hölder condition $^{4}$ on every compact subset of their domain of definition). Under this hypothesis it can be shown that the $(F, G)$ derivative of an $(F, G)$ pseudoanalytic function is continuous. Thus the class of $(F, G)$ pseudoanalytic functions of the second kind is the class of solutions $(\phi, \psi)$ of the (elliptic) system (3.2). It might be worthwhile to state explicitly that equation (3.2) can not be transformed, by a change of dependent and independent variables, into the Cauchy-Riemann system.

The proof of the continuity of the $(F, G)$ derivative is rather long and involves some delicate results from the theory of differential equations. A comparatively simpler proof can be given under the

4 The function $f(z)$ is said to satisfy a uniform Hölder condition if $\left|f\left(z_{1}\right)-f\left(z_{2}\right)\right|$ $\leqq K\left|z_{1}-z_{2}\right| \alpha$, for some $\alpha>0$ and $K$. 
additional hypothesis that the generators are Hölder continuously differentiable.

Together with the generating pair $(F, G)$ we consider the dual pair $(F, G)^{*}=\left(F^{*}, G^{*}\right)$ defined by the formulas

$$
F^{*}=\frac{2 \bar{G}}{F \bar{G}-\bar{F} G}, \quad G^{*}=\frac{2 \bar{F}}{F \bar{G}-\bar{F} G}
$$

(so that $F^{* *}=F, G^{* *}=G$ ). Equations (3.2), (3.3) imply the formulas

$$
2 \phi_{z}=F^{*} \dot{w}, \quad 2 \psi_{z}=-G^{*} \dot{w}
$$

which suggest the following definition of integration. Let $W(z)$ be a continuous function defined on a rectifiable curve $\Gamma$. The $(F, G)$ integral of $W$ on $\Gamma$ is, by definition,

$$
\int_{\Gamma} W d_{(F, G)} z=\operatorname{Re} \int_{\Gamma} F^{*} W d z-i \operatorname{Re} \int_{\Gamma} G^{*} W d z .
$$

$(F, G)$ derivatives may be characterized by the property that their $(F, G)$ integrals vanish along every closed curve contractible to a point in the domain considered. If $w(z)$ is $(F, G)$ pseudoanalytic of the first kind, then

$$
\omega\left(z_{1}\right)-\omega\left(z_{0}\right)=\int_{z_{0}}^{z_{1}} \dot{w} d_{(F, G) z}
$$

where $\omega=* w$ is the function of the second kind corresponding to $w$.

The most striking similarity between pseudoanalytic and analytic functions is revealed by the following theorem.

Let $(F, G)$ be a generating pair in a domain $D_{0}$. There exist in $D_{0}$ two other generating pairs, $\left(F_{1}, G_{1}\right)$ and $\left(F_{-1}, G_{-1}\right)$, called successor and predecessor of $(F, G)$, respectively, such that (i) $(F, G)$ derivatives of $(F, G)$ pseudoanalytic functions are $\left(F_{1}, G_{1}\right)$ pseudoanalytic of the first kind, and (ii) $(F, G)$ pseudoanalytic functions of the first kind are $\left(F_{-1}, G_{-1}\right)$ derivatives of $\left(F_{-1}, G_{-1}\right)$ pseudoanalytic functions. Conversely, $(F, G)$ is a successor of $\left(F_{-1}, G_{-1}\right)$ and a predecessor of $\left(F_{1}, G_{1}\right)$.

The actual construction of a successor pair in the large is a difficult task. In some cases it can be reduced to solving linear integral equations (for instance, if the domain $D_{0}$ is bounded and $F, G$ possess bounded derivatives with respect to $x$ and $y$ ). In the general case a more complicated limiting process is involved. Also, the successor pair $\left(F_{1}, G_{1}\right)$ is not determined uniquely; it may be replaced by any other equipotent pair. The construction of predecessors is reduced to the construction of successors, since it is not difficult to show that 
if $\left(F_{1}, G_{1}\right)$ is a successor of $(F, G),\left(F_{1}, G_{1}\right) *$ is a predecessor of $(F, G) *$. The theorem stated above implies that every given generating pair $(F, G)$ can be embedded in a generating sequence

$$
\cdots,\left(F_{-1}, G_{-1}\right),\left(F_{0}, G_{0}\right),\left(F_{1}, G_{1}\right),\left(F_{2}, G_{2}\right), \cdots
$$

that is in a sequence of generating pairs such that $\left(F_{0}, G_{0}\right)=(F, G)$ and $\left(F_{\nu+1}, G_{\nu+1}\right)$ is a successor of $\left(F_{\nu}, G_{\nu}\right)$ for all $\nu$.

A generating sequence $\left(F_{\nu}, G_{\nu}\right)$ is called periodic with period $N$ if $\left(F_{\nu+N}, G_{\nu+N}\right)=\left(F_{\nu}, G_{\nu}\right)$ for all $\nu$. The minimum period of a generating pair is the smallest period of a periodic generating sequence into which the pair can be embedded, if such a sequence exists, and is otherwise $\infty$. Recently Protter [74] succeeded in proving the existence of generating pairs with a prescribed minimum period $N$ (including the case $N=\infty)$.

It is easy to see that a generating pair $(F, G)$ is its own successor, i.e. has minimum period 1 , if and only if $(F / G)_{x} \equiv 0$. If $(F / G)_{x} \neq 0$ but $(F / G)_{y} \equiv 0$, the minimum period of $(F, G)$ is $2 .^{5}$

Now let $w(z)$ again be an $(F, G)$ pseudoanalytic function. Using a generating sequence $(3.8)$ in which $(F, G)$ is embedded we can define the higher derivatives of $w$ by the recursion formulas

$$
w^{[0]}=w ; \quad w^{[n+1]}=\frac{d_{\left(F_{n}, G_{n}\right) w^{[n]}}}{d z}, \quad n=1,2, \cdots .
$$

Thus a pseudoanalytic function of the first kind is, in a certain sense, infinitely differentiable, though as a function of the real variables $x$ and $y$ it need not have partial derivatives even of the first order.

4. Behavior at a point. $[11 ; 18]$. In describing the analytic properties of $(F, G)$ pseudoanalytic functions it is best to work with functions of the first kind. Let $w(z)=\phi F+\psi G$ be such a function. Then, in the neighborhood of every point of its domain of regularity we have (4.1) $w(z)=\phi\left(z_{0}\right) F(z)+\psi\left(z_{0}\right) G(z)+\alpha\left(z-z_{0}\right)^{n}+o\left(\left|z-z_{0}\right|^{n}\right), \alpha \neq 0$

where $\alpha$ is a complex constant, and $n$ a positive integer, except if $\dot{w} \equiv 0$ in which case $w \equiv \phi\left(z_{0}\right) F(z)+\psi\left(z_{0}\right) G(z)$. In particular, the zeros of a pseudoanalytic function are isolated, unless the function vanishes identically. This implies the unique continuation property $:{ }^{6}$ a pseudo-

${ }^{5} \mathrm{Cf}$., in this connection, Lukomskaya $[53 ; 54 ; 55]$.

- For differentiable $F, G$ this follows also from Carleman's theorem. Concerning various proofs of this theorem cf. $[12 ; 15 ; 36 ; 56 ; 81 ; 82]$. For a more general result, see $\$ 15$. 
analytic function is determined by its values on every open set, or even on every infinite set which has an accumulation point within the domain of regularity.

Now let $z_{0}$ be an isolated singular point of a single-valued pseudoanalytic function $w$. If $w(z) \rightarrow \infty$ as $z \rightarrow z_{0}, z_{0}$ is called a pole. In this case there exists an integer $n>0$ such that

$$
w(z)=\frac{\alpha}{\left(z-z_{0}\right)^{n}}+o\left(\left|z-z_{0}\right|^{-n}\right), \quad \alpha \neq 0 .
$$

We call $z_{0}$ an essential singular point of $w$ if $w(z)$ comes arbitrarily close to every complex number as $z \rightarrow z_{0}$. An isolated singularity of $a$ single-valued pseudoanalytic function is either removable, or a pole, or essential.

In view of the conformal covariance of pseudoanalyticity we can extend these results to finitely-many-valued functions. In fact, if $w(z)$ is $(F, G)$ pseudoanalytic for, say, $0<|z|<r$, and is $m$-valued, then $w\left(z^{m}\right)$ is a single-valued pseudoanalytic function with respect to the generators $F\left(z^{m}\right), G\left(z^{m}\right)$. Hence, if $w(z)$ remains bounded as $z \rightarrow 0$ we have

(4.3) $w(z)=\phi\left(z_{0}\right) F(z)+\psi\left(z_{0}\right) G(z)+\alpha\left(z-z_{0}\right)^{n / m}+o\left(\left|z-z_{0}\right|^{n / m}\right), \quad \alpha \neq 0$

where $n$ is a positive integer. Similarly, if $|w(z)| \rightarrow \infty$ for $z \rightarrow z_{0}$, we have the asymptotic formula

$$
w(z)=\frac{\alpha}{\left(z-z_{0}\right)^{n / m}}+o\left(\left|z-z_{0}\right|^{-n / m}\right), \quad \alpha \neq 0 .
$$

The asymptotic relations stated above can be "differentiated," as in the case of analytic functions. More precisely, if the (single-valued) $(F, G)$ pseudoanalytic function $w(z)$ has an essential singularity at $z_{0}$ so does the $(F, G)$ derivative $\dot{w}(z)$. If $w(z)$ is regular at $z_{0}$ and (4.1) holds, then

$$
\dot{w}(z)=n \alpha\left(z-z_{0}\right)^{n-1}+o\left(\left|z-z_{0}\right|^{n-1}\right) .
$$

If $z_{0}$ is a pole of $w(z)$ and (4.2) holds, we have

$$
\dot{w}(z)=-\frac{n \alpha}{\left(z-z_{0}\right)^{n+1}}+o\left(\left|z-z_{0}\right|^{-n-1}\right) .
$$

Similar conclusions can be drawn in the case of "algebraic" singularities: relation (4.3) implies that

$$
\dot{w}(z)=(n \alpha / m)\left(z-z_{0}\right)^{(n / m)-1}+o\left(\left|z-z_{0}\right|^{(n / m)-1}\right)
$$


and relation (4.4) that

$$
\dot{w}(z)=-\frac{n \alpha}{m\left(z-z_{0}\right)^{(n / m)+1}}+o\left(\left|z-z_{0}\right|^{-(n / m)-1}\right) .
$$

These results are easily extendable to pseudoanalytic functions defined in a neighborhood of the point at infinity.

In view of relations (4.1) and (4.2) we may speak of the order of a zero or a pole of a pseudoanalytic function, and the so called argument principle holds for pseudoanalytic functions of the first kind. In general, however, functions of the first kind do not share the geometric properties of analytic functions. This is clear from the fact that the generators themselves, which are essentially arbitrary functions, are pseudoanalytic. Geometric properties of pseudoanalytic functions are therefore to be discussed in terms of functions of the second kind.

The basic fact here is the following corollary of the asymptotic formulas (4.1), (4.1a). The mapping $\omega=\omega(z)$ by a pseudoanalytic function of the second kind $\omega=*$ w is a homeomorphism of a neighborhood of $a$ point $z_{0}$ if and only if $\dot{w}\left(z_{0}\right) \neq 0$. If $\dot{w}(z)$ has at $z_{0}$ a zero of order $n$, then $\omega(z)$ maps a suitably chosen neighborhood of $z_{0}$ onto an $(n+1)$ times covered neighborhood of $\omega\left(z_{0}\right)$. A similar statement can be made concerning points at which the function has poles.

Since $\dot{w}(z)$ is $\left(F_{1}, G_{1}\right)$ pseudoanalytic its zeros are isolated, and the pseudoanalytic function of the second kind $\omega(z)$ is a local homeomorphism everywhere except at isolated points. This local statement can be transformed into a global one via the general uniformization theorem: every pseudoanalytic function of the second kind is an interior transformation, that is it can be transformed into an analytic function by a homeomorphism of its domain of definition. It follows that the maximum principle holds for the modulus of a pseudoanalytic function $\omega=\phi+i \psi$ and also for the functions $\phi, \psi$.

The eccentricity of a differentiable mapping at a point at which the Jacobian is different from zero is defined as the eccentricity of the infinitesimal ellipse into which the mapping takes an infinitesimal circle. A direct computation shows that the eccentricity of a pseudoanalytic function can be estimated in terms of a bound for $|F|,|G|$ and $1 / \operatorname{Im}(\bar{F} G)$. Thus pseudoanalytic functions of the second kind are not only interior but also, at least on every compact subset of the domain in which the generators are defined, of bounded eccentricity (or quasi-conformal). This implies, among other things, that Picard's theorem on essential singularities holds. 


\section{Pseudoanalytic functions in the large}

5. Univalent functions. Local formal powers. The Riemann mapping theorem holds for pseudoanalytic functions of the second kind. This follows from a more general theorem (due to Miss Z. Sapiro) ${ }^{7}$ which asserts that the Riemann mapping theorem is true even for solutions of certain quasi-linear systems of elliptic partial differential equations. In proving the Riemann mapping theorem, however, one must make some assumptions, albeit very mild ones, on the behavior of the generators near the boundary of the domain considered (uniform boundedness of $|F|,|G|$ and $1 / \operatorname{Im}(\bar{F} G)$ is sufficient). On the other hand, if we do not want to solve a definite mapping problem, but merely find some univalent pseudoanalytic function, no hypotheses whatsoever on the domain or on the generators are required. As a matter of fact, the following is true $[13 ; 18]$.

Let the generators $(F, G)$ be defined in a domain $D_{0}$. Then there exist in $D_{0}$ two $(F, G)$ pseudoanalytic functions of the first kind, $w_{1}(z)$ and $w_{2}(z)$, such that for every choice of real constants $\lambda_{1}, \lambda_{2}$ with $\lambda_{1}^{2}+\lambda_{2}^{2}>0$, the function $\omega(z)=*\left(\lambda_{1} w_{1}+\lambda_{2} w_{2}\right)$ is univalent in $D_{0}$.

For the case of analytic functions (that is, for the generators $1, i$ ) the assertion is completely trivial if $D_{0}$ is a domain in the plane, since it suffices to set $w_{1}=z, w_{2}=i z$. But the assertion is true even if $D_{0}$ is a Riemann surface of genus zero, and in this case it is the (distinctly nontrivial) general uniformization theorem. The theorem stated above (for any pair of generators) is therefore a natural extension of the general uniformization theorem to pseudoanalytic functions.

This theorem is used in order to prove the existence of successors. Indeed, since $*\left(\lambda_{1} w_{1}+\lambda_{2} w_{2}\right)$ is univalent if $\lambda_{1}^{2}+\lambda_{2}^{2} \neq 0$, we have, noting the local univalency criterion stated in $\$ 4$, that $\operatorname{Im}\left(\left[\dot{w}_{1}\right]-\dot{w}_{2}\right) \neq 0$, everywhere. ${ }^{8}$ Hence we may assume that $\operatorname{Im}\left(\left[\dot{w}_{1}\right]-\dot{w}_{2}\right)>0$ renaming if necessary the functions $w_{1}, w_{2}$. It can be shown that $(F, G)$ derivatives are Hölder continuous (if the generators are). Hence we may define a pair of generators by setting $F_{1}=\dot{w}_{1}, G_{1}=\dot{w}_{2}$. It turns out that the pair $\left(F_{1}, G_{1}\right)$ is a successor of $(F, G)$.

In classical function theory the variable $z$ plays a double role: it is the independent variable and also the simplest nonconstant analytic function defined everywhere. In the case of an arbitrary pair of

7 [76]. An independent proof for linear systems was given by Gergen and Dressel $[36 ; 37 ; 38]$, a strengthened version of Šapiro's result by Nirenberg and the author [24]. Lavrent'ev proved a Riemann mapping theorem for general nonlinear systems $[49 ; 50]$.

$8[\dot{w}]-$ stands for the complex conjugate of $\dot{w}$. 
generators the independent variable $z$ is not pseudoanalytic, but we have just seen how one can find pseudoanalytic functions which have an important property (univalency) in common with the analytic functions $z, i z$. This suggests a way of constructing other pseudoanalytic functions which play the part of powers of the complex variable.

We assume now that the domain $D_{0}$ is simply connected and that a generating sequence $\left\{\left(F_{\nu}, G_{v}\right)\right\}$ is defined in $D_{0}$. We define the local formal power (with center at $z_{0}$ in $D_{0}$, coefficient $\alpha$ and exponent 0 ), $Z_{\nu}^{(0)}\left(\alpha, z_{0} ; z\right)$, as the linear combination of the generators $F_{\nu}(z), G_{\nu}(z)$ with real constant coefficients $\lambda, \mu$ chosen so that $\lambda F\left(z_{0}\right)+\mu G\left(z_{0}\right)=\alpha$. Thus $Z_{\nu}^{(0)}$ is determined by the conditions

$$
Z_{\nu}^{(0)}\left(\alpha, z_{0} ; z_{0}\right)=\alpha, \quad * Z_{\nu}^{(0)}\left(\alpha, z_{0} ; z\right)=\text { const. }\left(\bmod F_{\nu}, G_{\nu}\right) .
$$

The local formal powers with exponents $n=1,2, \cdots$ are defined by the recursion formulas

$$
{ }_{*} Z_{\nu}^{(n)}\left(\alpha, z_{0} ; z\right)=n \int_{z_{0}}^{z} Z_{\nu+1}^{(n-1)}\left(\alpha, z_{0} ; \zeta\right) d_{\left(F_{\nu}, G_{\nu}\right)} \zeta
$$

where we agree once and for all that ${ }_{*} Z_{\nu}$ is formed modulo $F_{\nu}, G_{\nu}$. We shall of ten write $F, G, Z$ instead of $F_{0}, G_{0}, Z_{0}$.

This definition implies the following properties. (i) $Z_{\nu}^{(n)}\left(\alpha, z_{0} ; z\right)$ is an $\left(F_{\nu}, G_{\nu}\right)$ pseudoanalytic function (of the first kind) of $z$. (ii) If $\alpha^{\prime}$ and $\alpha^{\prime \prime}$ are real constants, then

$$
Z_{\nu}^{(n)}\left(\alpha^{\prime}+i \alpha^{\prime \prime}, z_{0} ; z\right)=\alpha^{\prime} Z_{\nu}^{(n)}\left(1, z_{0} ; z\right)+\alpha^{\prime \prime} Z_{\nu}^{(n)}\left(i, z_{0} ; z\right) .
$$

(iii) The formal powers satisfy the differential relations

$$
\frac{d_{\left(F_{\nu}, G_{\nu}\right)}}{d z} Z_{\nu}^{(n)}\left(\alpha, z_{0} ; z\right)=n Z_{\nu+1}^{(n-1)}\left(\alpha, z_{0} ; z\right) .
$$

(iv) The asymptotic formulas

$$
Z_{p}^{(n)}\left(\alpha, z_{0} ; z\right) \sim \alpha\left(z-z_{0}\right)^{n},
$$$$
z \rightarrow z_{0}
$$

hold.

Assume now that

$$
w(z)=\sum_{n=0}^{\infty} Z^{(n)}\left(\alpha_{n}, z_{0} ; z\right)
$$

where the series converges uniformly in some neighborhood of $z_{0}$. 
It can be shown that the uniform limit of pseudoanalytic functions is pseudoanalytic, and that a uniformly convergent series of $(F, G)$ pseudoanalytic functions can be $(F, G)$ differentiated term by term. Hence the function $w$ in $(5.6)$ is $(F, G)$ pseudoanalytic and its $r$ th derivative admits the expansion

$$
w^{[r]}(z)=\sum_{n=r}^{\infty} n(n-1) \cdots(n-r+1) Z_{r}^{(n-r)}\left(\alpha_{n}, z_{0} ; z\right) .
$$

From this we obtain the Taylor formulas for the coefficients

$$
\alpha_{n}=\frac{w^{[n]}\left(z_{0}\right)}{n !} \text {. }
$$

Now let $w(z)$ be a given $(F, G)$ pseudoanalytic function defined for small values of $\left|z-z_{0}\right|$. The series

$$
\sum_{n=0}^{\infty} Z^{(n)}\left(\alpha_{n}, z_{0} ; z\right)
$$

with the coefficients given by (5.8) is called the Taylor series of $w$ at $z_{0}$, formed with local formal powers. Noting formulas (4.1), (4.1a), (5.5) we conclude that the Taylor series always represents the function asymptotically:

$$
w(z)-\sum_{n=0}^{N} Z^{(n)}\left(\alpha_{n}, z_{0} ; z\right)=O\left(\left|z-z_{0}\right|^{N+1}\right), \quad z \rightarrow z_{0},
$$

for all $N$. This implies (since a pseudoanalytic function can not have a zero of arbitrarily high order without vanishing identically) that the sequence of derivatives $\left\{w^{[n]}\left(z_{0}\right)\right\}$ determines the function w uniquely.

By the same token, if the series (5.9) converges uniformly in a neighborhood of $z_{0}$, it converges to the function $w$. Unfortunately the necessary and sufficient conditions for convergence are not known. A general expansion theorem holds for the global formal powers which are considered below.

The convergence question does not arise and the expansion (5.6) is certainly valid if all but a finite number of Taylor coefficients (5.8) vanish. This is the case if $w$ is a formal power with center $z_{1}$. Thus we have the formula

$$
Z^{(n)}\left(\alpha, z_{1} ; z\right)=\sum_{\mu=0}^{n}\left(\begin{array}{l}
n \\
\mu
\end{array}\right) Z^{(\mu)}\left[Z_{\mu}^{(n-\mu)}\left(\alpha, z_{1} ; z_{0}\right), z_{0} ; z\right]
$$

which generalizes the binomial theorem. 
6. Functions on a sphere. Global formal powers. $[11 ; 12]$. In this section we consider a generating pair $(F, G)$ defined on the full function-theoretical plane (Riemann sphere). By this we mean that the functions $F(z), G(z)$ are defined, are Hölder continuous and satisfy inequality (2.1) for all values of $z$, that the limits $F(\infty), G(\infty)$ exist, and that the generating pair $F(1 / z), G(1 / z)$ has similar properties. It now makes sense to talk about $(F, G)$ pseudoanalytic functions which are regular at $z=\infty$ or have a pole there. A pseudo-analytic function having no singularities, except perhaps poles, in the whole function theoretical plane will be called rational. The interiority of functions of the second kind implies that every rational pseudoanalytic function of the second kind can be transformed into a rational analytic function by a homeomorphism of the finite plane onto itself, the homeomorphism being dependent on the function considered.

A regular pseudoanalytic function defined in the whole finite plane is called entire. A bounded entire pseudoanalytic function of the first (second) kind is a linear combination of the generators with constant coefficients (a constant). This extension of Liouville's theorem shows that a rational pseudoanalytic function $w$ must have poles, except in the trivial case $* w=$ const. $A$ rational pseudoanalytic function has as many zeros as it has poles (zeros and poles being counted with their proper multiplicities).

A generating pair $(F, G)$ defined on the Riemann sphere has a successor $\left(F_{1}, G_{1}\right)$ and a predecessor $\left(F_{-1}, G_{-1}\right)$ defined on the Riemann sphere, and these pairs are determined uniquely (except, of course, for equivalences). It follows that $(F, G)$ can be embedded in an essentially unique generating sequence $\left\{\left(F_{\nu}, G_{\nu}\right)\right\}$ defined on the Riemann sphere. I conjecture that the sequence $\left\{\left(F_{\nu}, G_{v}\right)\right\}$ is nonperiodic unless $(F, G)$ is equivalent to $(1, i)$.

We are now in a position to define global formal powers with positive and negative exponents. The local formal powers formed with respect to the generating sequence described above are called global formal powers. The global formal power $Z_{\nu}^{(n)}\left(\alpha, z_{0} ; z\right), n>0$, is a rational $\left(F_{\nu}, G_{\nu}\right)$ pseudoanalytic function of $z$ which has a zero of order $n$ at $z=z_{0}$, a pole of order $n$ at $z=\infty$ and satisfies relation (5.5). An $\left(F_{v}, G_{v}\right)$ pseudoanalytic rational function $w(z)$ which has a pole of order $n$ at $z=z_{0} \neq \infty$, satisfies the asymptotic relation $w \sim \alpha\left(z-z_{0}\right)^{-n}$, $z \rightarrow z_{0}$, has a zero of order $n$ at $z=\infty$, and has no other zeros or poles, we call a global formal power with exponent $(-n)$, and denote by $Z_{v}^{(-n)}\left(\alpha, z_{0} ; z\right)$. The uniqueness of $Z^{(-n)}$ is easily established. In order to prove its existence it suffices to recall the conformal covariance of 
pseudoanalyticity. Define the generating pair $\left(\Phi_{\nu}, \Gamma_{\nu}\right)$, on the Riemann sphere, by setting

$$
\Phi_{\nu}(z)=F_{\nu}\left(z_{0}+1 / z\right), \quad \Gamma_{\nu}(z)=G_{\nu}\left(z_{0}+1 / z\right)
$$

and denote the $\left(\Phi_{\nu}, \Gamma_{\nu}\right)$ global formal powers (with positive exponents) by $\widehat{Z}_{\nu}^{(n)}$. For every $n>0$ and $\alpha$ one can find a number $\beta$ such that $\widehat{Z}_{\nu}^{(n)}(\beta, 0 ; z) \sim \alpha z^{n}, z \rightarrow \infty$. Then

$$
Z_{\nu}^{(-n)}\left(\alpha, z_{0} ; z\right)=\widehat{Z}_{\nu}^{(n)}\left(\beta, 0 ; \frac{1}{z-z_{0}}\right) .
$$

The properties (i)-(iv) of formal powers noted in $\$ 5$ remain valid for global formal powers with negative exponents.

Every rational pseudoanalytic function is a sum of formal powers. There exist rational pseudoanalytic functions with prescribed zeros and poles. Every pseudoanalytic function $w(z)$ which has a pole at $z_{0} \neq \infty$ is of the form

$$
w(z)=\sum_{\nu=1}^{N} Z^{(-n)}\left(\alpha_{n}, z_{0} ; z\right)+w_{1}(z), \quad \alpha_{N} \neq 0,
$$

where $w_{1}$ is pseudoanalytic and regular at $z_{0}$. An analogous decomposition into a "critical" and a regular part holds for functions which have a pole at infinity. Finally, there exist rational pseudoanalytic functions which have prescribed poles with prescribed critical parts.

The most important application of formal powers with negative exponents is the Cauchy integral formula. We first define a new kind of line integral for formal powers. Let $C$ be a continuously differentiable curve given by the equation $\zeta=\zeta(s), 0 \leqq s \leqq l, s$ being the arc length. If $g(\zeta)$ is a continuous function defined on $C$, we set

$$
\int_{C} Z^{(n)}[g(\zeta) d \zeta, \zeta ; z]=\int_{0}^{l} Z^{(n)}\left\{g[\zeta(s)] \zeta^{\prime}(s), \zeta(s) ; z\right\} d s .
$$

This integral as a function of $z$ is pseudoanalytic, everywhere if $n \geqq 0$, and everywhere except on $C$ if $n<0$. The definition is legitimate since $Z^{(n)}\left(\alpha, z_{0} ; z\right)$ can be shown to depend continuously on $z_{0}$.

Now if $w(z)$ is single-valued $(F, G)$ pseudoanalytic of the first kind in a domain $D$ interior to a sufficiently smooth curve $C$, and is continuous on $C$, then

$$
w(z)=\frac{1}{2 \pi} \int_{C} Z^{-1}[i w(\zeta) d \zeta, \zeta ; z], \quad z \text { in } D,
$$

and 


$$
\frac{1}{2 \pi} \int_{C} Z^{-1}[i w(\zeta) d \zeta, \zeta ; z]=0, \quad z \text { exterior to } D \text {. }
$$

A continuous function satisfying either of these relations for all $C$ is $(F, G)$ pseudoanalytic.

The Cauchy integral formula (6.5) can be used to derive reciprocity relations for formal powers. In the case of analytic functions it is trivial that the function $\alpha /\left(z-z_{0}\right)^{n}$ is an analytic function of $z_{0}$ as well as of $z$, but there exists a similar nontrivial statement about analytic functions on Riemann surfaces of higher genus (the theorem on interchanging parameter and argument in an Abelian integral of the second kind). In the case of pseudoanalytic functions the situation is nontrivial already on the sphere. Together with the generating sequence $\left\{\left(F_{\nu}, G_{v}\right)\right\}$ we consider the sequence of dual pairs $\left(F_{\nu}, G_{\nu}\right)^{*}$. (We noted before that $\left(F_{\nu}, G_{\nu}\right) *$ is a successor of $\left(F_{\nu+1}\right.$, $\left.G_{\nu+1}\right)^{*}$.) Let us denote the $\left(F_{\nu}, G_{\nu}\right)^{*}$ pseudoanalytic global formal powers by $Z_{\nu}^{*(n)}$. Then

$$
\begin{aligned}
& \operatorname{Re} Z_{\nu}^{(-n)}\left(1, z_{0} ; z\right)=(-1)^{n} \operatorname{Im} Z_{\nu-n}^{*(-n)}\left(i, z ; z_{0}\right), \\
& \operatorname{Re} Z_{\nu}^{(-n)}\left(i, z_{0} ; z\right)=(-1)^{n} \operatorname{Re} Z_{\nu-n}^{*(-n)}\left(i, z ; z_{0}\right), \\
& \operatorname{Im} Z_{\nu}^{(-n)}\left(1, z_{0} ; z\right)=(-1)^{n} \operatorname{Im} Z_{\nu-n}^{*(-n)}\left(1, z ; z_{0}\right), \\
& \operatorname{Im} Z_{\nu}^{(-n)}\left(i, z_{0} ; z\right)=(-1)^{n} \operatorname{Re} Z_{\nu-n}^{*(-n)}\left(1, z ; z_{0}\right),
\end{aligned}
$$

for $n=1,2, \cdots$. The corresponding reciprocity relations for powers with positive exponents read

$$
\begin{aligned}
& \operatorname{Re} Z_{\nu}^{(n)}\left(1, z_{0} ; z\right)=(-1)^{n} \operatorname{Re} Z_{\nu+n}^{*(n)}\left(1, z ; z_{0}\right), \\
& \operatorname{Re} Z_{\nu}^{(n)}\left(i, z_{0} ; z\right)=-(-1)^{n} \operatorname{Im} Z_{\nu+n}^{*(n)}\left(1, z ; z_{0}\right), \\
& \operatorname{Im} Z_{\nu}^{(n)}\left(1, z_{0} ; z\right)=-(-1)^{n} \operatorname{Re} Z_{\nu+n}^{*(n)}\left(i, z ; z_{0}\right), \\
& \operatorname{Im} Z_{\nu}^{(n)}\left(i, z_{0} ; z\right)=(-1)^{n} \operatorname{Im} Z_{\nu+n}^{*(n)}\left(i, z ; z_{0}\right)
\end{aligned}
$$

for $n=0,1,2, \cdots$. These relations hold also for local formal powers.

The global formal powers with integral exponents correspond to Abelian integrals of the second kind. In order to construct logarithmic pseudoanalytic functions, corresponding to Abelian integrals of the third kind on the sphere, we may use either of the two integration processes at our disposal. More precisely, set

$$
\left.L_{\nu}\left(\alpha, z_{0} ; z_{1}, z_{2} ; z\right)=\frac{1}{2 \pi} \int_{g}^{z_{2}} Z_{\nu}^{(-1)}\left\{i Z_{\nu}^{(0)}\left(\alpha, z_{0} ; \zeta\right) d \zeta, \zeta ; z\right)\right\}
$$

where $z_{1} \neq z_{2}$ and the integration is performed along some curve join- 
ing these two points. Considered as a function of $z, L_{v}$ is a multiplevalued $\left(F_{v}, G_{v}\right)$ pseudoanalytic function, which increases by

$$
(r-s) Z_{\nu}^{(0)}\left(\alpha, z_{0} ; z\right)
$$

when $z$ goes $r$ times around $z_{1}$ and $s$ times around $z_{2}$. On the other hand, the $\left(F_{v}, G_{v}\right)$ pseudoanalytic function (of the first kind) of $z$, $\Lambda_{v}\left(\alpha, z_{0} ; z_{1} ; z\right)$ which is defined by setting

$$
{ }_{*} \Lambda_{\nu}\left(\alpha, z_{0} ; z_{1} ; z\right)=\frac{1}{2 \pi} \int_{1+z_{1}}^{z} Z_{\nu+1}^{(-1)}\left\{Z_{\nu}^{(0)}\left(\alpha, z_{0} ; z_{1}\right), z_{1} ; \zeta\right\} d_{\left(F_{\nu}, G_{\nu}\right)},
$$

possesses logarithmic singularities at $z_{1}$ and at $z=\infty$ and increases by $Z_{\nu}^{(0)}\left(\alpha, z_{0} ; z\right)$ when $z$ goes once around $z_{1}$. These two functions are connected by the relation

$$
L_{\nu}\left(\alpha, z_{0} ; z_{1}, z_{2} ; z\right)=\dot{\Lambda}_{\nu}\left(\alpha, z_{0} ; z_{1} ; z\right)-\dot{\Lambda}_{\nu}\left(\alpha, z_{0} ; z_{2} ; z\right)
$$

where the dot indicates $\left(F_{\nu}, G_{v}\right)$ differentiation.

The simplest finitely-many-valued pseudoanalytic functions are the global formal powers with fractional exponents. These are defined with the aid of the generating pairs $\left(\Phi_{v}, \Gamma_{v}\right)$,

$$
\Phi_{\nu}(z)=F_{\nu}\left(z_{0}+z^{m}\right), \quad \Gamma_{\nu}(z)=G_{\nu}\left(z_{0}+z^{m}\right)
$$

where $m$ is an integer, $m>1$. We denote the $\left(\Phi_{\nu}, \Gamma_{\nu}\right)$ pseudoanalytic global formal powers with integral exponents by $\widehat{Z}_{\nu}^{(n)}$. If $r$ is a positive or negative integer, relatively prime to $m$, then the $\left(F_{\nu}, G_{\nu}\right)$ pseudoanalytic formal power with exponent $r / m$ is defined by setting

$$
Z_{\nu}^{(r / m)}\left(\alpha, z_{0} ; z\right)=Z_{\nu}^{(r)}\left(\alpha, 0 ;\left(z-z_{0}\right)^{1 / m}\right) .
$$

The properties (i)-(iv) of formal powers noted in $\$ 5$ remain valid also for powers with fractional exponents.

The global formal powers with fractional exponents are the simplest examples of "algebraic" pseudoanalytic functions. In general, a multiple-valued $(F, G)$ pseudoanalytic function will be called algebraic if it is finitely-many-valued and can be continued along every path in the finite plane avoiding a finite number of excluded "critical" points, provided that at every critical point as well as at the point at infinity the function becomes infinite of at most a finite order. An analytic function satisfying these conditions is algebraic in the ordinary sense, that is, a solution of an algebraic equation the coefficients of which are rational functions. For pseudoanalytic algebraic functions only a weakened form of this statement is true. At all but a finite number of points a pseudoanalytic algebraic function takes on 
the same finite number of distinct values. The sum of these values is called the trace of the function. It is not difficult to prove that the trace of an algebraic pseudoanalytic function is a rational pseudoanalytic function. In particular, the trace of a formal power $Z^{(n)}\left(\alpha, z_{0} ; z\right)$, with $n$ not an integer, is zero.

7. Doubly-periodic pseudoanalytic functions. [20]. A generating pair $(F, G)$ is called doubly periodic if the functions $F(z)$ and $G(z)$ possess two periods $\Omega_{1}$ and $\Omega_{2}$, with $\operatorname{Im}\left(\bar{\Omega}_{1} \Omega_{2}\right)>0$. An $(F, G)$ pseudoanalytic function which has the same periods and is regular everywhere except for poles may be called an $(F, G)$ elliptic function. An $(F, G)$ elliptic function of the second kind can be transformed into an ordinary (analytic) elliptic function by a homeomorphism of the plane. Different $(F, G)$ elliptic functions, however, will, in general, be transformed into analytic elliptic functions with different periods. The classical existence theorems of the theory of elliptic functions remain valid, when properly restated, in the pseudoanalytic case.

In particular, there exist, in analogy to the Weierstrass $\zeta$-function, two linearly independent everywhere regular $(F, G)$ pseudoanalytic functions, $\zeta_{1}(z)$ and $\zeta_{2}(z)$, such that for all integers $m, n$

$$
\zeta_{j}\left(z+m \Omega_{1}+n \Omega_{2}\right)-\zeta_{j}(z)=m \lambda_{j} F(z)+n \mu_{j} G(z), \quad j=1,2,
$$

the constants $\lambda_{j}, \mu_{j}$ being real. The derivatives $\dot{\zeta}_{1}(z), \dot{\zeta}_{2}(z)$ have, therefore, the periods $\Omega_{1}, \Omega_{2}$, and it can be shown that if we set $F_{1}=\dot{\zeta}_{1}$, $G_{1}=\dot{\zeta}_{2}$ (interchanging, if necessary, the functions $\zeta_{1}, \zeta_{2}$ ), we obtain a generating pair $\left(F_{1}, G_{1}\right)$ which is a successor to $(F, G)$. It follows that $(F, G)$ can be embedded in a generating sequence $\left\{\left(F_{\nu}, G_{\nu}\right)\right\}$ such that all functions $F_{\nu}(z), G_{\nu}(z)$ have the periods $\Omega_{1}, \Omega_{2}$. The pairs $\left(F_{\nu}, G_{\nu}\right)$ are determined uniquely, except for equivalences.

I conjecture that if such a sequence satisfies the relation $\left(F_{n}, G_{n}\right)$ $=\left(F_{0}, G_{0}\right)$, then either $n=1$ or $n=2$ (that these two cases are indeed possible can be shown by simple examples).

8. Pseudoanalytic functions and differentials on Riemann surfaces. [20]. Let $S$ be an abstract Riemann surface. Two functions $F, G$ defined on $S$ form a generating pair if they are Hölder continuous in terms of local parameters and satisfy the relation $\operatorname{Im}(\bar{F} G)>0$. A function $w$ defined on $S$ will be called an $(F, G)$ function if in the neighborhood of each point $p$ of $S$ and for every local parameter $z$ defined in such a neighborhood, $w$ is an $(F, G)$ pseudoanalytic function of $\boldsymbol{z}$ which has no singularities except poles. A differential $d W$ defined on $S$ will be called an $(F, G)$ differential if for every local parameter $z$ defined in a sufficiently small neighborhood of a point 
$p$ of $S$ there exists an $(F, G)$ function $w(z)$ defined in the neighborhood such that $d W=\dot{w} d z$. Every $(F, G)$ function gives rise to an $(F, G)$ differential, but an $(F, G)$ differential is the differential of an $(F, G)$ function only if all its $(F, G)$ periods vanish, the $(F, G)$ period of $d W$ over a closed curve $\Gamma$ being defined as the integral

$$
\int_{\mathrm{r}} \operatorname{Re}\left(F^{*} d W\right)-i \operatorname{Re}\left(G^{*} d W\right) .
$$

The $(F, G)$ period of an $(F, G)$ differential $d W$ over a small simple closed curve enclosing a singular point $p$ of $d W$ is called the residue of $d W$ at $p$.

A multiple-valued $(\widetilde{F}, \widetilde{G})$ pseudoanalytic function $w(z)$ defined in a subdomain $\Delta$ of the full function-theoretical plane may be considered as a single-valued $(F, G)$ pseudoanalytic function defined on a suitably chosen covering surface $S$ of $\Delta$. The generators $F, G$ are obtained from $\tilde{F}, \widetilde{G}$ by transplantation: if the point $p$ of $S$ covers the point $z$ of $\Delta$, then $F(p)=\widetilde{F}(z), G(p)=\widetilde{G}(z)$. In particular, if $\Delta$ is the whole Riemann sphere and the function $w$ is algebraic, the surface $S$ is closed (compact). The converse statements, however, are not generally true, unlike the classical situation. In fact, while an abstract Riemann surface $S$ carrying a generating pair $(F, G)$ can be represented, in infinitely many ways, as a covering surface of a domain $\Delta$ on the Riemann sphere, one can not, in general, choose this representation so that the generators $F, G$ are obtained by transplantation from a generating pair defined in $\Delta$.

Uniformization of multiple-valued pseudoanalytic functions (or, more generally, of functions on a Riemann surface) is accomplished by the usual procedure: construction of the universal covering surface, and conformal mapping on a standard domain $\Sigma$ which is either the full function-theoretical plane, or the finite plane, or the unit disc. The result reads: every multiple-valued pseudoanalytic function $w(z)$ admits the parametric representation

$$
w=\widehat{w}(\tau), \quad z=\widehat{z}(\tau)
$$

where $\tau$ ranges over $\Sigma, \hat{z}(\tau)$ is analytic and $\widehat{w}(\tau)$ is $(\widehat{F}, \widehat{G})$ pseudoanalytic, the functions $\widehat{F}(\tau), \widehat{G}(\tau), \widehat{z}(\tau), \widehat{w}(\tau)$ being automorphic with respect to the same properly discontinuous group of fixed point free conformal mappings of $\Sigma$ onto itself.

We consider next $(F, G)$ functions and differentials on a closed Riemann surface $S$ of genus $g$. The cases $g=0$ and $g=1$ have already been considered in the preceding two sections. If $g>1$, then there 
exists no preferred parametrization and it makes no sense to talk about the successor of $(F, G)$ on $S$.

Using the interior character of pseudoanalytic functions of the second kind it is easy to show that an $(F, G)$ function (which is not a linear combination of the generators with real constant coefficients) has as many zeros as it has poles, and that an $(F, G)$ differential (which does not vanish identically) has $2(g-1)$ more zeros than poles, zeros and poles being counted with their multiplicities. A similar argument shows that the sum of the residues of an $(F, G)$ differential vanishes, and that an everywhere regular $(F, G)$ differential which does not vanish identically has a nonreal $(F, G)$ period.

The existence theorem for $(F, G)$ differentials states that there are exactly $2 g$ linearly independent, everywhere regular $(F, G)$ differentials, and that the number of linearly independent $(F, G)$ differentials which have poles of order not exceeding $\beta_{1}, \cdots, \beta_{r}$ at $r>1$ distinct points $p_{1}, \cdots, p_{r}$ is $2\left(g-1+\beta_{1}+\cdots+\beta_{r}\right)$. This implies that one may prescribe the real parts of the periods of an $(F, G)$ differential $d w$ along $2 g$ homologically independent closed curves as well as the singularities of the differential, provided one chooses these singularities in such a way that the sum of the residues vanishes.

In order that an $(F, G)$ differential which has poles at $r>1$ distinct points be the differential of an $(F, G)$ function, its $r$ residues and its periods on $2 g$ homologically independent closed curves must vanish. This amounts to not more than $2(g+r-1)$ linear (real) conditions. Hence the existence theorem for $(F, G)$ differentials also implies the existence of nontrivial $(F, G)$ functions.

The Riemann-Roch theorem for pseudoanalytic functions involves the dual generating pair. Let $A$ be the number of linearly independent $(F, G)$ functions which have zeros at $r$ distinct points $p_{1}, \cdots, p_{r}$ of orders at least $\alpha_{1}, \cdots, \alpha_{r}$, and poles at most at $s$ distinct points $q_{1}, \cdots, q_{s}$, of orders not exceeding $\beta_{1}, \cdots, \beta_{s}$, and let $B$ be the number of linearly independent $(F, G)^{*}$ differentials which have no poles except perhaps at the points $p_{j}$, of orders not exceeding $\alpha_{j}$, and which have zeros at the points $q_{k}$ of orders at least $\beta_{k}$. Set $m=\beta_{1}+\cdots+\beta_{\text {s }}$ $-\alpha_{1}-\cdots-\alpha_{r}$. Then

$$
A-B=2(m+1-g) .
$$

In particular, if $m>2 g-2$ then $B=0$ and therefore $A=2(m+1-g)$.

While the Riemann-Roch theorem can be stated in terms of divisors it should be remembered that the $(F, G)$ functions on $S$ do not form a field. As for the proof of the theorem, it depends on reciprocity relations between $(F, G)$ differentials and $(F, G) *$ differentials, of which relations (6.6) are a typical though very special sample. 


\section{DiffERENTIABLE GENERATORS AND THE SIMILARITY PRINCIPLE}

9. Differentiable generators. $[11 ; 12 ; 15 ; 81 ; 82]$. We return to single-valued pseudoanalytic functions defined in a plane domain $D_{0}$. The theory acquires new features if we assume that in $D_{0}$ the generators possess partial derivatives with respect to the real variables $x$ and $y$. In this case we can define the "characteristic coefficients" $a(z), b(z), A(z), B(z)$ of the pair $(F, G)$. These are determined by the formulas

$$
\begin{array}{ll}
F_{\bar{z}}=a F+b \bar{F}, & G_{z}=a G+b \bar{G}, \\
F_{z}=A F+B \bar{F}, & G_{z}=A G+B \bar{G} .
\end{array}
$$

A simple computation shows that the existence of the $(F, G)$ derivative $\dot{w}$ implies the equations

$$
\begin{aligned}
w_{z} & =a w+b \bar{w}, \\
\dot{w} & =w_{z}-A w-B \bar{w} .
\end{aligned}
$$

Another generating pair $\left(F_{1}, G_{1}\right)$ having the characteristic coefficients $a_{1}, b_{1}, A_{1}, B_{1}$ will be a successor of $(F, G)$ if and only if

$$
a_{1}=a, \quad b_{1}=-B .
$$

Equivalent generating pairs have the same characteristic coefficients; for equipotent generating pairs the first two characteristic coefficients ( $a$ and $b$ ) coincide.

Assume now, for the sake of orientation, that the domain $D_{0}$ is bounded and that the characteristic coefficients are Hölder continuous and bounded. Then the following theorems hold.

(i) A function $w(z)$ is $(F, G)$ pseudoanalytic of the first kind if and only if it satisfies equation (9.3).

(ii) $A$ bounded continuous function $w(z)$ defined in $D_{0}$ is $(F, G)$ pseudoanalytic of the first kind if and only if the function $h(z)$ defined by the equation

$$
w(z)=-\frac{1}{\pi} \iint_{D_{0}} \frac{a(\zeta) w(\zeta)+b(\zeta) \overline{w(\zeta)}}{\zeta-z} d \xi d \eta+h(z)
$$

is analytic.

We note that if the boundary $C_{0}$ of $D_{0}$ is sufficiently smooth and $w$ is continuous on $C_{0}$ the function $h$ in (9.6) admits the representation

$$
h(z)=\frac{1}{2 \pi i} \int_{c_{0}} \frac{h(\zeta) d \zeta}{\zeta-z} .
$$


Equation (9.6) may be considered as an integral equation for the unknown function $w(z)$.

(iii) Equation (9.6) has a unique solution w for every bounded continuous $h$.

This last theorem is due to Vekua [81] who also investigated in detail the resolvent of equation (9.6). ${ }^{9}$

The hypotheses under which we stated these theorems can be considerably relaxed. First of all, there is no need to assume that the partial derivatives of $F$ and $G$ (or, what is the same, the characteristic coefficients) are Hölder continuous, though if one drops this assumption the derivatives occurring in equations (9.1)-(9.4) must be understood in some generalized sense. Vekua considered the case of continuous $a, b$. The continuity assumption, however, is still too restrictive, and has the disadvantage of being nonhereditary. If the generators $F, G$ have continuous partial derivatives, the same is not necessarily true for other $(F, G)$ pseudoanalytic functions of the first kind; and the continuity of $a, b$ does not insure the continuity of $A, B$. A sufficient and hereditary smoothness condition is the following: the characteristic coefficients $a, b$ are measurable and are absolutely integrable to a power $p>2$ in the neighborhood of every point of $D_{0}$. An example (due to Soboleff and published by Vekua [83]) shows that the condition $p>2$ can not be weakened.

The assumption that the domain and the characteristic coefficients be bounded is also too severe. Let $\rho(z)$ be a measurable function defined in the whole plane such that $|\rho(z)|{ }^{p}$ is locally integrable for some $p>1$. We call $\rho$ admissible if there exist constants $M>0$ and $\epsilon$, $0<\epsilon<1$ such that

$$
\begin{gathered}
\iint \frac{|\rho(\zeta)| d \xi d \eta}{|\zeta-z|} \leqq \frac{M}{1+|z|^{1+\epsilon}}, \\
\iint \frac{|\rho(\zeta)| d \xi d \eta}{\left|\zeta-z_{1}\right|\left|\zeta-z_{2}\right|} \leqq \frac{M}{\left|z_{1}-z_{2}\right|^{1-\epsilon}},
\end{gathered}
$$

the integration being performed over the whole plane. A function $\rho(z)$ defined in a domain $D_{0}$ is called admissible if it becomes so upon setting $\rho=0$ in the complement of $D_{0}$.

If $\rho(z)$ vanishes outside a large circle, it is admissible if and only if there exists a $K>0$ such that

$$
\iint_{|\zeta-z|<r<1}|\rho(\zeta)| d \xi d \eta \leqq K r^{2-\epsilon}
$$

- Vekua assumed some regularity of the boundary of $D$. For the general case see I. Polonsky's forthcoming thesis. 
A sufficient condition (Vekua) is that $\rho$ belong to $L_{p}, p>2$.

Theorems (i)-(iii) are valid if the characteristic coefficients $a, b$ are admissible.

The following result [15] shows that we could have defined pseudoanalyticity by means of equation (9.3).

(iv) If the functions $a(z), b(z)$ are admissible in $D_{0}$, there exists $a$ generating pair $(F, G)$ in $D_{0}$ for which $a$ and $b$ are the first two characteristic coefficients.

The proof of (iv) depends on the similarity principle stated below.

10. Similarity principle. Let $(F, G)$ be a generating pair defined in a domain $D_{0}$. Under certain conditions the following theorem $[10$; $11 ; 81 ; 84]$ holds for every subdomain $D \subset D_{0}$.

(v) (Similarity principle for a general domain). (a) Let w(z) be a single-valued $(F, G)$ pseudoanalytic function of the first kind defined in a domain $D$. Then there exists an analytic function $f(z)$, and a complexvalued function $s(z)$ defined on the closure of $D$ and having $a$ bound and $a$ Hölder modulus of continuity depending only on $(F, G)$, such that

$$
w(z)=e^{s(z)} f(z) .
$$

(b) Let $f(z)$ be a single-valued analytic function defined in a domain $D$. There exists a function $s(z)$ satisfying the conditions stated above such that the function $w(z)$ defined by $(10.1)$ is $(F, G)$ pseudoanalytic.

A sufficient condition for the validity of the similarity principle is the existence of admissible characteristic coefficients $a, b$. The proof of statement (a) is then almost trivial, since the function $s$ can be written down explicitly (modulo an analytic function):

$$
s(z)=\frac{1}{\pi} \iint_{D}\left\{a(\zeta)+b(\zeta) \frac{\overline{w(\zeta)}}{w(\zeta)}\right\} \frac{d \xi d \eta}{\zeta-z} .
$$

To prove statement (b) one may consider the nonlinear integral equation which must be satisfied (modulo an analytic function) by the function $s$. It reads

$$
s(z)=\frac{1}{\pi} \iint_{D}\left\{a(\zeta)+b(\zeta) \overline{e^{s(\zeta)}-8(\zeta)} \frac{\overline{f(\zeta)}}{f(\zeta)}\right\} \frac{d \xi d \eta}{\zeta-z} .
$$

Since $s$ enters into the right hand side only in the form $e^{\bar{s}-s}$ it is easy to show, using the Schauder fixed point theorem, that this equation is solvable. Another way to prove statement (b) is to consider the linear integral equation, similar to equation (9.6), which must be satisfied by the function $e^{s}$. 
Under the same conditions we can prove:

(vi) (Similarity principle with a boundary condition) [15]. Assume that the boundary of $D$ contains a simple closed Lyapounoff curve ${ }^{10} C$. Then theorem (iv) remains valid if one requires that $s(z)$ be real on $C$ and vanish at a given point on $C$.

An extension of this theorem to the case of several contours $C$ is possible if one considers not single-valued functions but functions with a single-valued argument (Lee [51]).

The similarity principle may fail for a trivial reason, since it may happen that one of the generators, say $F(z)$, can not be represented in the form $e^{s} f$, with a bounded $s$ and an analytic $f$. It is an important open problem to find necessary and sufficient conditions for the validity of the similarity principle.

Partial extensions of part (a) of the similarity principle to the case of nonadmissible coefficients have been considered by Vekua [84] and by C. Morawetz. ${ }^{11}$ A refinement of the similarity principle in the case in which the behaviour of the characteristic coefficients is known at a point is due to $\mathrm{N}$. Newman. ${ }^{11} \mathrm{~A}$ quite different and far-reaching extension of statement (a) occurs in the theory of elliptic equations with discontinuous coefficients developed by Nirenberg and the writer (cf. $\$ 15$ below).

Assume now that for the generators considered the similarity principle holds. Then we can at once transfer a large number of classical function-theoretical results to the case of pseudoanalytic functions. Thus, according to statement (b), there exist in every domain pseudoanalytic functions with prescribed zeros and poles, and every domain is an existence domain for a pseudoanalytic function which can not be continued into any larger domain. Using statement (a), on the other hand, we can extend to pseudoanalytic functions such theorems on bounded analytic functions as Jensen's inequality, Blashke's theorem on the location of zeros, Fatou's theorem on the existence of radial limits almost everywhere, the uniqueness theorems of $F$. and M. Riesz and of Privaloff and Lusin, etc. The similarity principle with a boundary condition may be used for the solution of boundary value problems, for the construction of Green and Neumann functions, etc.

The most striking application of the similarity principle, however, is to global formal powers and power series.

\footnotetext{
${ }^{10}$ A Lyapounoff curve is a curve $z=z(t)$ with $z^{\prime}(t) \neq 0$ and $z^{\prime}(t)$ satisfying a uniform Hölder condition.

11 To appear.
} 
11. Expansion and approximation theorems. In this section we consider a generating pair $(F, G)$ defined on the Riemann sphere for which the similarity principle holds. This condition will be satisfied, for instance, if the functions $F(z), G(z), F(1 / z), G(1 / z)$ have continuous partial derivatives for $|z|<+\infty$. We consider the global formal powers ${ }^{12}$ and apply to them part (a) of the similarity principle. Noting relation (5.5) and the fact that $Z^{(n)}\left(\alpha, z_{0} ; z\right)$ is $O\left(|z|^{n}\right)$ at infinity we conclude that $Z^{(n)}\left(\alpha, z_{0} ; z\right)=e^{s(z)} \beta\left(z-z_{0}\right)^{n}$ where the constant $\beta$ is such that $e^{s\left(z_{0}\right)} \beta=\alpha$. Hence

$$
e^{-2 K} \leqq\left|\frac{Z^{(n)}\left(\alpha, z_{0} ; z\right)}{\alpha\left(z-z_{0}\right)^{n}}\right| \leqq e^{2 K}
$$

where $K$ is the bound on $|s|$ which depends only on $(F, G)$. This inequality shows that the global formal power series

$$
\sum_{n=0}^{\infty} Z^{(n)}\left(\alpha_{n}, z_{0} ; z\right)
$$

converges within the circle of convergence of the ordinary power series $\sum \alpha_{n}\left(z-z_{0}\right)^{n}$ and diverges outside of this circle. Moreover, we have the following theorem (due to Agmon and the author [1]).

If $w(z)$ is an $(F, G)$ pseudoanalytic function defined for $\left|z-z_{0}\right|<r$ $\leqq+\infty$, then the Taylor series of $w$ formed with global formal powers $Z^{(n)}\left(\alpha, z_{0} ; z\right)$ converges (and hence represents the function) for $\left|z-z_{0}\right|<r$.

We shall sketch the proof as an example of the application of the similarity principle. Consider first the special function

$$
w(z)=Z^{(-1)}(\beta, \zeta ; z)
$$

and denote its Taylor coefficients at a point $z_{0} \neq \zeta$ by $\alpha_{n}$. According to (5.4) and (5.8) we have

$$
\alpha_{n}=(-1)^{n} Z_{n}^{(-1-n)}\left(\beta, \zeta ; z_{0}\right),
$$

but here we make no use of these relations. For every $n$ the function

$$
W_{n}(z)=Z^{(-1)}(\beta, \zeta ; z)-\sum_{\mu=0}^{n-1} Z^{(\mu)}\left(\alpha_{\mu}, z_{0} ; z\right)
$$

is a rational $(F, G)$ pseudoanalytic function which has no poles except at $z=\zeta$ and $z=\infty$, and satisfies the relations

12 Under the hypotheses stated above global formal powers can be constructed' independently of the procedure described in $\$ \$ 5,6$, by means of part (b) of the similarity principle. 


$$
\begin{array}{cc}
W_{n}(z) \sim \beta(z-\zeta)^{-1}, z \rightarrow \zeta ; \quad W_{n}(z) \sim \alpha_{n}\left(z-z_{0}\right)^{n}, & z \rightarrow z_{0} ; \\
W_{n}(z)=O\left(|z|^{n}\right), & z \rightarrow \infty .
\end{array}
$$

By the similarity principle we have

$$
W_{n}(z)=e^{s(z)} \gamma \frac{\left(z-z_{0}\right)^{n}}{z-\zeta}, \quad \gamma=\text { const. }
$$

where $|s| \leqq K$, and using the first two relations (11.6) we conclude that $\beta=\gamma e^{s(\zeta)}\left(\zeta-z_{0}\right)^{n}, \alpha_{n}=\gamma e^{s\left(z_{0}\right)}\left(z_{0}-\zeta\right)^{-1}$, so that

$$
\left|\alpha_{n}\right| \leqq e^{2 K}|\beta|\left|\zeta-z_{0}\right|^{-n-1}, \quad n=0,1, \cdots .
$$

Thse inequalities imply that the expansion

$$
Z^{(-1)}(\beta, \zeta ; z)=\sum_{n=0}^{\infty} Z^{(n)}\left(\alpha_{n}, z_{0} ; z\right)
$$

converges for $\left|z-z_{0}\right|<\left|\zeta-z_{0}\right|$. The expansion theorem being established for the case of the special function (11.3), the transition to arbitrary functions is accomplished, just as in the classical case, by means of the Cauchy integral formula (6.4) applied to a circle.

Similar arguments yield the more general Laurent expansion.

Let $w(z)$ be a single-valued $(F, G)$ pseudoanalytic function defined in the domain

$$
0 \leqq r<\left|z-z_{0}\right|<R \leqq \infty
$$

Then

$$
w(z)=\sum_{n=-\infty}^{+\infty} Z^{(n)}\left(\alpha_{n}, z_{0} ; z\right)
$$

the series being uniformly and absolutely convergent in every annulus

$$
r<r^{\prime} \leqq\left|z-z_{0}\right| \leqq R^{\prime}<R \text {. }
$$

As a corollary we obtain an expansion theorem for multiple-valued functions.

If $w(z)$ is an m-valued $(F, G)$ pseudoanalytic function defined in the domain (11.10), then it admits in (11.10) the unique expansion

$$
w(z)=\sum_{n=-\infty}^{+\infty} Z^{(n / m)}\left(\alpha_{n}, z_{0} ; z\right) .
$$

Finally, the expansion theorem in conjunction with the Cauchy formula yields the analogue of Runge's theorem [12].

$A$ single-valued $(F, G)$ pseudoanalytic function defined in a domain $D$ 
can be expanded into a series of rational pseudoanalytic functions which converges uniformly and absolutely on every compact subset of $D$. If $D$ is simply connected, w can be expanded into a series of formal polynomials (entire rational pseudoanalytic functions).

Summarizing we see that in the case of sufficiently smooth generators $(F, G)$, defined over the Riemann sphere, $(F, G)$ pseudoanalytic functions can be characterized by each of the following properties: (i) having $(F, G)$ derivatives, (ii) being $\left(F_{-1}, G_{-1}\right)$ derivatives, (iii) being $(F, G)$ integrals of $\left(F_{1}, G_{1}\right)$ pseudoanalytic functions, (iv) satisfying the generalized Cauchy-Riemann equation (3.2), (v) satisfying the generalized Cauchy-Riemann equation (9.3) (vi) obeying the generalized Cauchy formula (6.4) or (6.5), (vii) admitting an expansion in a formal power series in every disc of regularity, (viii) being the uniform limit of a sequence of formal polynomials in every simply connected domain of regularity. The analogy with classical function theory is now complete.

(Added in proof (August 2, 1956). For generators $(F, G)$ defined on the Riemann sphere one can prove, without assuming differentiability or the similarity principle, that the expansion (11.2) converges and represents the function for $\left|z-z_{0}\right|<\theta r$, where $\theta>0$ is a constant depending only on $(F, G)$. The proof [88] depends on the reciprocity relations (6.6) and on the Calderón-Zygmund theory of singular integrals [89]. Instead of the inequalities (11.1) one has now the weaker inequalities

$$
e^{-|n| K} \leqq\left|\frac{Z^{(n)}\left(\alpha, z_{0}: z\right)}{\alpha\left(z-z_{0}\right)^{n}}\right| \leqq e^{|n| K}
$$

where the constants $K_{\nu}$ depend on $(F, G)$ and on $\nu$.)

\section{Applications}

12. Reduction of linear elliptic equations to pseudoanalytic functions. In two dimensions every harmonic function can be expressed in terms of analytic functions of a complex variable, in two ways. If $h(x, y)$ satisfies Laplace's equation, then it is the real part of an analytic function $f(z)$. Also, the complex gradient $g(z)=h_{x}-i h_{y}$ is analytic and $g(z)=f^{\prime}(z)$. A similar relation exists between pseudoanalytic functions and solutions of linear elliptic equations of second order, subject to very mild smoothness assumptions $[11 ; 12 ; 15 ; 19]$.

We assume at first that all equations considered have Hölder continuous coefficients.

(a) The simplest linear elliptic equation is of the form 


$$
\phi_{x x}+\phi_{y y}+A_{1} \phi_{x}+A_{2} \phi_{y}=0 .
$$

Assume that the coefficients $A_{1}, A_{2}$ are admissible in the domain considered (cf. §9). Using Theorem (iv) we can find functions $\sigma>0$ and $\tau$ such that (12.1) can be obtained by elimination from the elliptic system

$$
\phi_{x}=\tau \psi_{x}+\sigma \psi_{y}, \quad \phi_{y}=-\sigma \psi_{x}+\tau \psi_{y} .
$$

This system may be written in the form (3.2) with

$$
F=1, \quad G=-\tau+i \sigma .
$$

Thus every solution $\phi$ of $(12.1)$ is the real part of an $(F, G)$ pseudoanalytic function of the second kind, and vice versa. On the other hand, the complex gradient of $\phi, W=\phi_{x}-i \phi_{y}$ satisfies the equation

$$
W_{\bar{z}}=a_{1} W+\bar{a}_{1} \bar{W}, \quad a_{1}=-\left(A_{1}+i A_{2}\right) / 4 .
$$

By Theorem (iv) there exists a generating pair $\left(F_{1}, G_{1}\right)$ such that every solution of this equation is $\left(F_{1}, G_{1}\right)$ pseudoanalytic of the first kind. Finally, the $(F, G)$ derivative of the $(F, G)$ function $w=\phi+\psi G$ is

$$
\dot{w}=\phi_{z}+\psi_{z} G=W .
$$

Hence $\left(F_{1}, G_{1}\right)$ is a successor of $(F, G)$.

For further reference we note that system (12.1) may also be written in the form

$$
\omega_{\bar{z}}=\nu \bar{\omega}_{\bar{z}}
$$

where $\omega=\phi+i \psi$ and

$$
\nu=-(F+i G) /(F-i G)
$$

so that $|\nu|<1$.

(b) If the equation

$$
A_{11} \phi_{x x}+2 A_{12} \phi_{x y}+A_{22} \phi_{y y}=0
$$

is elliptic, we may assume without loss of generality that

$$
A_{11} A_{22}-A_{12}^{2} \equiv 1, \quad A_{11}>0 .
$$

Consider the Riemann metric

$$
A_{22} d x^{2}-2 A_{12} d x d y+A_{11} d y^{2} .
$$

There exists a homeomorphism $\zeta=\zeta(z)=\xi+i \eta$ of the domain of definition of (12.5) which is conformal with respect to the metric (12.7), that is a solution of the Beltrami equation 


$$
\zeta_{z}=\widehat{\mu} \zeta_{z}
$$

where

$$
\mu=\frac{A_{22}-A_{11}-2 i A_{12}}{A_{22}+A_{11}+2} .
$$

If the coefficients $A_{i j}$ are Hölder continuously differentiable, the functions $\xi$ and $\eta$ have Hölder continuous second derivatives and introducing them as new independent variables we transform equation (12.5) into the canonical form (12.1).

If the $A_{i j}$ are only Hölder continuous, the reduction to pseudoanalytic functions is still possible. On the one hand, we can find an elliptic system of the form

$$
\phi_{x}=b_{11} \psi_{x}+b_{12} \psi_{y}, \quad \phi_{y}=b_{21} \psi_{x}+b_{22} \psi_{y}
$$

which is equivalent to the single equation (12.5). On the other hand, the complex gradients $\phi_{x}-i \phi_{y}=U+i V$ of a solution of (12.5) satisfy the elliptic system

$$
A_{11} U_{x}=2 A_{12} V_{x}+A_{22} V_{y}, \quad U_{y}=-V_{x} .
$$

The transformation $\zeta=\zeta(z)$ takes both systems into equations of the form (12.4). Thus $\phi+i \psi$ and $\phi_{x}-i \phi_{y}$ are pseudoanalytic functions of $\zeta$ (with different generators).

(c) Solutions of an elliptic equation

$$
A_{11} \phi_{x x}+2 A_{12} \phi_{x y}+A_{22} \phi_{y y}+A_{1} \phi_{x}+A_{2} \phi_{y}=0,
$$

with $A_{i j}$ satisfying (12.6), can be expressed by pseudoanalytic functions if the coefficients $A_{i j}$ are Hölder continuously differentiable, for in this case the mapping $\zeta(z)$ described above takes (12.12) into an equation of the form (12.1).

If the coefficients are only Hölder continuous, the reduction is possible locally. In fact, in the neighborhood of any point we can transform equation (12.12) to the form (12.5) by introducing as new independent variables two solutions of (12.12).

(d) The most general linear homogeneous elliptic equation

$$
\text { (12.13) } A_{11} \phi_{x x}+2 A_{12} \phi_{x y}+A_{22} \phi_{y y}+A_{1} \phi_{x}+A_{2} \phi_{y}+A_{0} \phi=0
$$

can be transformed into an equation of the form (12.12) in every domain in which it has a positive solution $\phi_{0}$. This is accomplished by introducing the new unknown function $\phi / \phi_{0}$. A solution $\phi_{0}>0$ will exist in every small domain, and in every large domain if $A_{0} \leqq 0$.

(e) We consider next a first order elliptic system in normal form 


$$
u_{x}=\tau v_{x}+\sigma v_{y}+b_{11} u+b_{12} v, \quad-u_{y}=\sigma v_{x}-\tau v_{y}+b_{21} u+b_{22} v
$$

with $\sigma>0$. Setting

$$
u+i v=w,
$$

we write this system in the form

$$
w_{z}=v 2 \bar{w}_{z}+\alpha w+\beta w \bar{w}
$$

where $|\nu|<1$.

If $\alpha=\beta=0$, this equation shows that $w$ is a pseudoanalytic function of the second kind (cf. (a)). If $\alpha$ and $\beta$ do not vanish the reduction to pseudoanalytic functions is possible in the large if $\nu$ is Hölder continuously differentiable, and locally if $\nu$ is Hölder continuous.

In the first case set $W=w-\nu w$. Equation $\left(12.14^{\prime}\right)$ takes the form

$$
W_{i}=a W+b \bar{W} .
$$

Hence (cf. Theorem (iv) of $\S 9$ ) $W$ is a pseudoanalytic function of the first kind.

In the second case we can find near any given point two solutions $w_{1}$ and $w_{2}$ of $\left(12.14^{\prime}\right)$ such that $\operatorname{Im}\left(\bar{w}_{1} w_{2}\right)>0$. The new unknown function $W$ defined by the relation

$$
w=W\left(w_{1}-i w_{2}\right)+\bar{W}\left(w_{1}+i w_{2}\right)
$$

satisfies an equation of the form (12.16) and is therefore pseudoanalytic.

The general linear homogeneous system

$$
\begin{aligned}
u_{x} & =a_{11} v_{x}+a_{12} v_{y}+b_{11} u+b_{12} v, \\
-u_{y} & =a_{21} v_{x}+a_{22} v_{y}+b_{21} u+b_{22} v
\end{aligned}
$$

is called elliptic if

$$
4 a_{12} a_{21}-\left(a_{11}+a_{22}\right)^{2}>0, \quad \quad a_{12}>0 .
$$

In this case it is equivalent to a single complex equation for the function (12.15),

$$
w_{z}=\mu w_{z}+\nu \bar{w}_{z}+\alpha w+\beta \bar{w}
$$

with

$$
|\mu|+|\nu|<1 .
$$

Let $\zeta=\zeta(z)$ be a homeomorphism conformal with respect to the metric of (12.17), that is, a univalent solution of the Beltrami equation (12.8) with 


$$
\widehat{\mu}=\frac{a_{12}-a_{21}-i\left(a_{11}+a_{22}\right)}{a_{12}+a_{21}+\left[4 a_{12} a_{21}-\left(a_{11}+a_{22}\right)^{2}\right]^{1 / 2}} .
$$

The mapping $z \rightarrow \zeta$ takes equation $\left(12.17^{\prime}\right)$ into the canonical form $\left(12.14^{\prime}\right)$.

The preceding results may be extended by noting that there was no need to assume the coefficients $A_{1}, A_{2}, A_{0}, b_{i j}, \alpha, \beta$ to be Hölder continuous. It would suffice to require that they be measurable functions belonging to $L_{p}$ for some $p>2$. In this case the second derivatives in equations (12.1), (12.12) and (12.13) and the first derivatives in equations (12.14), (12.16) and (12.17) are to be understood in a generalized sense.

13. An example. Equations of mixed type. The concepts of $(F, G)$ differentiation and integration were first formulated, by Gelbart and the author $[21 ; 22]$, for systems of the special form

$$
\sigma(x) \phi_{x}=\tau(y) \psi_{y}, \quad \sigma(x) \phi_{y}=-\tau(y) \psi_{x} .
$$

Systems of this form occur frequently in mechanics of continua. For $\sigma=1, \tau=y^{p}$, one obtains the equations of Weinstein's generalized axially symmetric potential theory [85].

System (13.1) is equivalent to equation (3.2) for the generating pair

$$
F=\sigma(x)^{1 / 2} \tau(y)^{-1 / 2}, \quad G=i \sigma(x)^{-1 / 2} \tau(y)^{1 / 2}
$$

where we assume that $\sigma>0, \tau>0$. If $(\phi, \psi)$ is a solution of (13.1) we may form the functions

$$
\phi^{\prime}=\sigma \phi_{x}, \quad \psi^{\prime}=-\phi_{y} / \sigma
$$

and

$$
\text { (13.4) } \Phi=\int \sigma \phi d x-\tau \psi d y, \quad \Psi=\int(\psi / \sigma) d x+(\phi / \tau) d y,
$$

the path independence of the integrals being a consequence of (13.1). Clearly, $\left(\phi^{\prime}, \psi^{\prime}\right)$ and $(\Phi, \Psi)$ are solutions of the system

$$
\phi_{x} / \sigma(x)=\tau(y) \psi_{y}, \quad \phi_{y} / \sigma(x)=-\tau(y) \psi_{x} .
$$

In other words: if (13.1) holds, the function

$$
w=(\sigma / \tau)^{1 / 2} \phi+i(\tau / \sigma)^{1 / 2} \psi=\phi F+\psi G
$$

is $(F, G)$ pseudoanalytic, and its $(F, G)$ derivative

$$
\dot{w}=(\sigma / \tau)^{1 / 2}\left(\phi_{x}-i \phi_{y}\right)=\phi^{\prime} F_{1}+\psi^{\prime} G_{1}
$$

is $\left(F_{1}, G_{1}\right)$ pseudoanalytic, where 


$$
F_{1}=\sigma(x)^{-1 / 2} \tau(y)^{-1 / 2}, \quad G_{1}=i \sigma(x)^{1 / 2} \tau(y)^{1 / 2} .
$$

The function

$$
W=(\sigma \tau)^{-1 / 2} \Phi+i(\sigma \tau)^{1 / 2} \Psi=\Phi F_{1}+\Psi G_{1}
$$

is $\left(F_{1}, G_{1}\right)$ pseudoanalytic, and its $\left(F_{1}, G_{1}\right)$ derivative is $w$. Thus $\left(F_{1}, G_{1}\right)$ is a successor and a predecessor of $(F, G)$ and

$$
\cdots,\left(F_{1}, G_{1}\right), \quad(F, G), \quad\left(F_{1}, G_{1}\right), \quad(F, G), \cdots
$$

is a generating sequence.

The local formal powers determined by this sequence are easily found. For the sake of simplicity we put the center at the origin and set

$$
* Z^{(n)}(a, 0 ; z)=* Z^{(n)}(a, z), \quad(\bmod F, G) .
$$

Define the functions $X_{j}^{(n)}(x), Y_{j}^{(n)}(y), j=1,2 ; n=0,1, \cdots$ by the recurrence relations:

$$
\begin{gathered}
X_{j}^{(0)}=Y_{j}^{(0)} \equiv 1, \quad X_{j}^{(n)}(0)=Y_{j}^{(n)}(0)=0 \\
\frac{d X_{j}^{(n)}}{d x}= \begin{cases}n \sigma X_{j}^{(n-1)}, & n+j \text { even }, \\
n X_{j}^{(n-1)} / \sigma, & n+j \text { odd },\end{cases} \\
\frac{d Y^{(n)}}{d y}= \begin{cases}n \tau Y_{j}^{(n-1)}, & n+j \text { even }, \\
n Y_{j}^{(n-1)} / \tau, & n+j \text { odd } .\end{cases}
\end{gathered}
$$

Then

$$
{ }_{*} Z^{(n)}(\alpha+i \beta, z)=\sum_{\nu=0}^{n} i^{\nu}\left(\begin{array}{l}
n \\
\nu
\end{array}\right)\left[\alpha X_{k}^{(n-\nu)} Y_{l}^{(\nu)}+i \beta X_{l}^{(n-p)} Y_{k}^{(\nu)}\right]
$$

where $k=2, l=1$ if $n$ is odd and $k=1, l=2$ if $n$ is even.

Since equations (13.1) may be solved by separation of variables, functions pseudoanalytic with respect to the generators (13.2) have many properties not encountered in the general theory $[23 ; 9]$. Of principal importance is the fact that the formalism described above remains valid also for a system of the form

$$
\sigma_{1}(x) \phi_{x}=\tau_{1}(y) \psi_{x}, \quad \sigma_{2}(x) \phi_{y}=-\tau_{2}(y) \psi_{x}
$$

provided one replaces, in (13.3)-(13.6)

$$
\sigma \text { by } \sigma_{2}, \quad \frac{1}{\sigma} \text { by } \frac{1}{\sigma_{1}}, \quad \tau \text { by } \tau_{2}, \quad \frac{1}{\tau} \text { by } \frac{1}{\tau_{1}} .
$$

If the coefficients $\sigma_{1}, \sigma_{2}, \tau_{1}, \tau_{2}$ are positive, system (13.8) may be re- 
duced to the form (13.1) by changing independent variables. But the formalism works also for hyperbolic and elliptico-hyperbolic systems (13.8), that is, if some of the coefficients are negative or change sign. In the latter case, of course, some of the integrals defining the functions (13.6) may diverge.

We note in particular the system

$$
\phi_{x}=\psi_{y}, \quad \phi_{y}=-K(y) \psi_{x}, \quad y K(y)>0 .
$$

Elimination of $\phi$ leads to the elliptico-hyperbolic equation of TricomiChaplygin type

$$
K(y) \psi_{x x}+\psi_{y y}=0
$$

which is of paramount importance in transonic gas dynamics. The particular solutions (13.7) have been used in studying initial [10] and boundary value problems (Morawetz [58]) for equation (13.10). In the Tricomi case $(K(y)=y)$ these particular solutions are ordinary polynomials in $x$ and $y$.

14. Applications of pseudoanalytic functions. These applications are all based on the fact noted in $\$ 12$. In two dimensions the theory of second order elliptic equations with, say, Hölder continuous coeffcients (and of systems of two first order equations) is essentially identical with that of pseudoanalytic functions. ${ }^{13}$ Hence every result described in $\$ \S 2-11$ can be reformulated as a theorem on differential equations, in which the concept of pseudoanalyticity is not even mentioned. One obtains in this way very precise information on the nature of solutions defined in the whole plane, on zeros and singularities, on boundary behavior of solutions (Chang [30]) and on the uniqueness of solution of the Cauchy problem [15]. The similarity principle of $\$ 10$, which is a general structure and existence theorem, plays a decisive part in this and other applications; in particular, it leads to the construction of Green's functions [15] and analogues of harmonic measures (Lee [51]). The similarity principle and the integral equation (9.6) have been applied also to boundary value problems (Vekua [81], and others).

Even when the same result can be proved directly, the use of pseudoanalytic functions may be advantageous. For instance, it is known that solutions of elliptic equations may be expressed, using the so-called fundamental solution, in terms of Cauchy data on the boundary (Bergman [8], Položii [70], Sabat [75]). But our derivation

\footnotetext{
${ }^{13}$ On the other hand, the theory of pseudoanalytic functions has few points of contact with the methods of Bergman [7] and Vekua [80] who use integral operators to represent solutions of analytic elliptic equations.
} 
of the "Cauchy formula" (6.4) gives this representation under very weak hypotheses and, instead of relying on the existence of the fundamental solutions, yields a simple existence proof.

As another example we mention a lemma of $\mathrm{H}$. Lewy which has several applications in differential geometry in the large. Let $\phi(x, y)$ satisfy a nonlinear elliptic differential equation

$$
\Omega\left(x, y, \phi, \phi_{x}, \phi_{y}, \phi_{x x}, \phi_{x y}, \phi_{y y}\right)=0 .
$$

Assume that $\Omega(x, y, 0,0,0,0,0,0) \equiv 0$ and that $\phi$ vanishes at the origin together with its second derivatives. Then either $\phi$ is a linear function, or $\phi_{x y}^{2}-\phi_{x x} \phi_{y y}>0$ for $0<x^{2}+y^{2}<\epsilon$.

Lewy [52] proved this for an analytic equation, Hartman and Wintner [41] for $\Omega$ of class $C^{2}$. An application of pseudoanalytic functions yields the lemma under essentially sharp conditions: Hölder continuous differentiability of $\Omega$ [19].

There also exist other applications to nonlinear elliptic equations [14]. But the most interesting of these refer to the equations of gas dynamics.

The velocity potential $\phi$, stream-function $\psi$ and density $\rho$ of a twodimensional flow are connected by the equations

$$
\rho \phi_{x}=\psi_{y}, \quad \rho \phi_{y}=-\psi_{x}
$$

which show that $\phi+i(\psi / \rho)$ is an $(1, i / \rho)$ pseudoanalytic function of the first kind; the $(1, i / \rho)$ derivative of this function is the complex velocity $\phi_{x}-i \phi_{y}$. In gas dynamics one assumes $\rho$ to be a given function of $q=\left|\phi_{x}-i \phi_{y}\right|$. In this case equations (14.2) are nonlinear, but the hodograph transformation leads to a linear system of the form (13.1). Pseudoanalytic function techniques have been used in establishing the existence and uniqueness of subsonic flows past given profiles [16], the existence of compressible Helmholtz flows (Berg [5]), the Kutta-Joukowsky theorem for gas flows $\left(\mathrm{Newman}^{11}\right)$, and the infinitesimal nonexistence theorem for transonic flows (Morawetz [57]).

\section{Extensions}

15. Elliptic equations with discontinuous coefficients. While the theory of pseudoanalytic functions yields a complete description of elliptic equations (12.13) and elliptic systems (12.1) with smooth, say Hölder continuous, coefficients it is important, especially in view of nonlinear problems, to consider also equations with merely measurable coefficients.

System $\left(12.17^{\prime}\right)$ is called uniformly elliptic if the coefficients are measurable functions and there exists a constant $k$ such that 


$$
|\mu|+|\nu| \leqq k<1 \text {. }
$$

We also assume that

$$
|\alpha|+|\beta| \leqq k^{\prime}<+\infty,
$$

though this condition can be weakened. In general there will be no continuously differentiable solution. A solution $w=u+i v$ of $\left(12.17^{\prime}\right)$ is required only to be continuous and to have strong $L_{2}$ derivatives in the sense of Soboleff and Friedrichs. The basic result is the following representation theorem due to Nirenberg and the author [24].

Let $w(z)$ be a solution of $\left(12.17^{\prime}\right)$ defined in a subdomain $D$ of the unit disc. Then w(z) may be written in the form

$$
w(z)=e^{s(z)} f[x(z)]
$$

where $s(z)$ is continuous for $|z| \leqq 1$, real on $|z|=1$ and vanishes at $z=0, \zeta=\chi(z)$ is a homeomorphism of $|z| \leqq 1$ onto $|\zeta| \leqq 1$ such that $\chi(0)=0, \chi(1)=1$, and $f(\zeta)$ is analytic on $\Delta=\chi(D)$. The functions $s(z), \chi(z)$ and $z=\chi^{-1}(\zeta)$ satisfy uniform Hölder conditions depending only on $k$ and $k^{\prime}$.

If $\alpha \equiv \beta \equiv 0$, (15.3) holds with $s \equiv 0$. This result is contained in the work of Morrey [59]. If $\mu \equiv \nu \equiv 0,(15.2)$ holds with $\chi(z) \equiv z$, and we obtain the similarity principle of $\$ 9$.

The representation theorem implies that unless $w \equiv 0$, the zeros of $w(z)$ are isolated and not of infinite order. Thus solutions of (12.17) always have the unique continuation property. The representation (15.3) also permits us to classify isolated singularities into poles and essential singularities. But such precise asymptotic formulas as the ones given in $\S 4$, let alone generalized differentiation, are out of the question.

Equation (12.13) is called uniformly elliptic if the coefficients are measurable, satisfy (12.6), and are uniformly bounded (the latter condition can be weakened for the coefficients $A_{1}, A_{2}$ and $A_{0}$ ). If $\phi$ satisfies a uniformly elliptic equation (12.12), $W=\phi_{x}-i \phi_{y}$ satisfies a uniformly elliptic equation $\left(12.17^{\prime}\right)$ and obeys the representation theorem. This leads, in particular, to the strong maximum principle for equation (12.12), and also for equation (12.13) with $A_{0} \geqq 0$. (E. Hopf's well known proof [44] of the maximum principle does not work for discontinuous coefficients.)

The most important consequences of the representation theorem, however, are strong a priori estimates which permit one to treat boundary value problems for nonlinear elliptic equations [25]. 
16. Elliptic systems of order $2 m>2$ in two dimensions. Sobrero [77] observed that solutions of the biharmonic equation $\Delta^{2} \phi(x, y)=0$ can be expressed in terms of analytic functions on a certain algebra. More generally, solutions of $\Delta^{m} \phi(x, y)=0$ can be expressed in terms of analytic functions on an algebra $A_{m}$, an algebra over the real field generated by a unit $j$ such that $\left(1+j^{2}\right)^{m}=0$, which is isomorphic to the algebra over the complex field generated by a unit $\omega$ with $\omega^{m}=0$.

Diaz [31] considered pseudoanalytic functions of $x+j y$, with values in $A_{m}$, defined by certain special systems of $2 m$ first order equations for $2 m$ unknown functions. This system yields, for each function, an elliptic equation of the form

$$
L^{m} \phi=0,
$$

$L$ being an elliptic operator which may be reduced to the form

$$
L \phi=\Delta \phi+a(x) \phi_{x}+b(y) \phi_{y} .
$$

To these functions Diaz extended the formalism of $\$ 13$ (differentiation, integration, and formal powers and power series).

The algebra $A_{m}$ also plays a part in the more general investigations of Douglis $[32 ; 34]$. Douglis obtained a normal form for the general linear elliptic system of $2 m$ first order equations and pointed out a special cass of these systems, called generalized Beltrami systems, solutions of which can be expressed in terms of "hyper-analytic" functions. The theory of hyper-analytic functions bears a very close resemblance to classical function theory, since these functions themselves form an algebra. For $m=1$ one obtains ordinary Beltrami systems which can be transformed into the Cauchy-Riemann equations. It is remarkable that for $m>1$ a generalized Beltrami system can not, in general, be transformed into a system with constant coefficients.

Douglis also stated the problem of developing a theory of pseudoanalytic functions applicable to the general case (that is, to nonBeltrami systems). But this problem is still open. The chief obstacle is the lack of a Carleman type theorem asserting the nonexistence of zeros of infinite order. (The closely related but weaker theorem on the unique solvability of Cauchy's problem has been proved; cf. Carleman [29], Douglis [33], Hartman and Wintner [40].)

17. The higher-dimensional case. During the last years spectacular progress has been achieved in the theory of elliptic equations and systems of order $2 m \geqq 2$ in a space of $n>2$ dimensions. But as far as the deeper function-theoretical properties of solutions are concerned little is known, even in the classical case $2 m=2$. 
It is very likely that a solution of any homogeneous elliptic equation or system cannot vanish of infinite order at a point without vanishing identically. The statement is obvious for equations with analytic coefficients, since such equations have only analytic solutions (John [45]); the difficulty lies in the nonanalytic case. For $n=2$, $2 m=2$ the answer is known, as we have seen above. For $n>2$ this unique continuation theorem has been proved thus far (by Müller [64] and Heinz [43]) for equations of the form

$$
\Delta \phi+\sum_{i=1}^{n} a_{i} \frac{\partial \phi}{\partial x_{i}}+a_{0} \phi=0
$$

where $\Delta$ is the Euclidean Laplace operator.

(Added in proof (August 2, 1956). Aronszajn [87] proved the unique continuation theorem for second order elliptic equations with sufficiently smooth coefficients. Another proof is due to Cordes (to appear). The weaker form of the unique continuation theorem, i.e. the unique solvability of the Cauchy problem, was also proved (for $2 m=2, n>2$ ) by Landis [91] and by Pedersen (to appear). New proofs of the Müller-Heinz result were given by Hartman and Wintner [90] and by Lax (to appear). Nirenberg proved the uniqueness theorem for the Cauchy problem for certain equations with $2 m>2$ and $n \geqq 2$, under restrictive assumptions on the shape of the initial surface (to appear).)

About zeros of finite order there exists a rather general result [17]. If $\phi(x)=\phi\left(x_{1}, \cdots, x_{n}\right)$ satisfies an elliptic equation

$$
L \phi=\sum_{0 \leqq i_{1}+\cdots+i_{n} \leqq 2 m} a_{i_{1} \cdots i_{n}}(x) \frac{\partial i_{1}+\cdots+i_{n} \phi(x)}{\partial x_{1}^{i_{1}} \cdots \partial x_{n}^{i_{n}}}=0
$$

with Hölder continuous coefficients, and vanishes at $x=0$, but not of infinite order, then

$$
\phi(x)=p(x)+o\left(|x|^{N}\right)
$$

where $p(x)$ is a homogeneous polynomial of degree $N$ and $p(x) \not \equiv 0$. This polynomial satisfies the "osculating" equation with constant coefficients, $L_{0} p=0, L_{0}$ being obtained from $L$ by replacing $a_{i_{1}} \ldots i_{n}(x)$ by $a_{i_{1}} \cdots i_{n}(0)$ if $i_{1}+\cdots+i_{n}=2 m$ and by 0 otherwise.

If $\phi(x)$ is defined in a deleted neighborhood of the origin and $\phi(x)=O\left(|x|^{2 m-n}\right)$, then

$$
\phi(x)=c J(x)+o\left(|x|^{2 m-n}\right),
$$


$J(x)$ being a fundamental solution of the osculating equation. If $\phi(x)=o(|J(x)|)$, the singularity at $x=0$ is removable. Also, the asymptotic relations (17.3), (17.4) may be formally differentiated $2 m$ times. (Much more is known about singularities in the analytic case [45].)

The parallelism between these results and those described in $\$ 4$ suggests strongly that analogues of formal powers and corresponding expansion theorems could be found for general linear elliptic equations. For second order equations of the special form

$$
\Delta \phi+\sum_{i=1}^{n} a_{i}\left(x_{i}\right) \frac{\partial \phi}{\partial x_{i}}=0
$$

this was accomplished by Protter [74] several years ago. The general problem is open.

While the results described in this and the preceding sections are still rather episodic, they all point in the same direction-toward a general function theory of elliptic equations which will relegate pseudoanalytic functions to the position of a special case.

\section{BIBLIOGRAPHY}

1. S. Agmon and L. Bers, The expansion theorem for pseudoanalytic functions, Proc. Amer. Math. Soc. vol. 3 (1952) pp. 757-764.

2. L. Ahlfors, On quasi-conformal mappings, Journal d'Analyse Mathématique vol. 4 (1954) pp. 1-58.

3. E. Beltrami, Sulle funzioni potenziali di sistemi simmetrici intorno ad un asse, Opere matematice, Vol. 3, Milano, 1911, pp. 115-128.

4. - Sulle teoria delle funzioni potenziali simmetriche, ibid., pp. 349-377.

5. P. W. Berg, On the existence of Helmholtz flows of a compressible fluid, Dissertation, New York University, 1953.

6. S. Bergman, Functions satisfying certain partial differential equations of elliptic type and their representation, Duke Math. J. vol. 14 (1947) pp. 349-366.

7. - Linear operators in the theory of partial differential equations, Trans. Amer. Math. Soc. vol. 53 (1943) pp. 130-155.

8. - A formula for the streamfunction of certain flows, Proc. Nat. Acad. Sci. U. S. A. vol. 29 (1943) pp. 276-281.

9. L. Bers, The expansion theorem for sigma-monogenic functions, Amer. J. Math. vol. 72 (1950) pp. 705-712.

10. - On the continuation of a potential gas flow across the sonic line, N.A.C.A. Technical Note No. 2058, 1950.

11. - Partial differential equations and generalized analytic functions, Proc. Nat. Acad. Sci. U.S.A. vol. 36, (1950) pp. 130-136; Second note, ibid., vol. 37 (1951) pp. 42-47.

12. - Theory of pseudo-analytic functions, Lecture notes (mimeographed), New York University, 1953.

13. - Univalent solutions of linear elliptic systems, Comm. Pure Appl. Math. vol. 6 (1953) pp. 513-526. 
14. - Nonlinear elliptic equations without nonlinear entire solutions, Journal of Rational Mechanics and Analysis vol. 3 (1954) pp. 767-787.

15. - Function-theoretical properties of solutions of partial differential equations of elliptic type, Annals of Mathematics Studies, no. 33, 1954, pp. 69-94.

16. - Existence and uniqueness of a subsonic flow past a given profile, Comm. Pure Appl. Math. vol. 7 no. 3 (1954) pp. 441-504.

17. - Local behavior of solutions of general linear elliptic equations, Comm. Pure Appl. Math. vol. 8, no. 4 (1955).

18. - Local theory of pseudoanalytic functions, Lectures on functions of a complex variable, Michigan University Press, 1955, pp. 213-244.

19. - Remark on an application of pseudoanalytic functions, Amer. J. Math. vol. 78 (1956) pp. 486-496.

20. - Partial differential equations and pseudoanalytic functions on Riemann surfaces, Annals of Mathematics Studies, no. 30, 1953, pp. 157-165.

21. L. Bers and A. Gelbart, On a class of differential equations in mechanics of continua, Quarterly of Applied Mathematics vol. 1 (1943) pp. 168-188.

22. - On a class of functions defined by partial differential equations, Trans. Amer. Math. Soc. vol. 56 (1944) pp. 67-93.

23. - On generalized Laplace transformations, Ann. of Math. vol. 48 (1947) pp. $342-357$.

24. L. Bers, and L. Nirenberg, On a representation theorem for linear elliptic systems with discontinuous coefficients and its applications, Convegno Internazionale sulle Equazioni Derivate e Parziali, Agosto, 1954, pp. 111-140.

25. - On linear and nonlinear elliptic boundary value problems in the plane, Convegno Internazionale sulle Equazioni Derivate e Parziali, Agosto, 1954, pp. 141-167.

26. W. M. Boothby, The topology of the level curves of harmonic functions with critical points, Amer. J. Math. vol. 73 no. 3 (1951) pp. 512-538.

27. R. Cacciopoli, Fondamenti per una teoria generale delle funzioni pseudoanalitiche di una variabile complessa, Academia nazionale dei Lincei, Rendiconti vol. 13 (1952) pp. 197-204 and 321-329.

28. T. Carleman, Sur les systèmes linéaires aux dérivées partielles du premier ordre à deux variables, C. R. Acad. Sci. Paris vol. 197 (1933) pp. 471-474.

29. - Sur un problème d'unicité pour les systèmes d'équations aux dérivées partielles à deux variables indépendentes, Arkiv för Matematik, Astronomi och Fysik vol. 26B No. 17 (1939) pp. 1-9.

30. H. H. Chang, Approximately analytic functions of bounded type and boundary behavior of solutions of elliptic partial differential equations, Acta Math. Sinica vol. 3 (1953) pp. 101-132.

31. J. B. Diaz, On a class of partial differential equations of even order, Amer. J. Math. vol. 68 (1946) pp. 611-659.

32. A. Douglis, $A$ function-theoretic approach to elliptic systems of equations in two variables, Comm. Pure Appl. Math. vol. 6 (1953) pp. 259-289.

33. - Uniqueness in Cauchy problems for elliptic systems of equations, Comm. Pure Appl. Math. vol. 6 (1953) pp. 291-298.

34. - Function-theoretic properties of certain elliptic systems of first order equations, Lectures on Functions of a Complex Variable, Michigan University Press, 1953, pp. 335-340.

35. A. Gelbart, On a function-theory method for obtaining potential flow patterns of a compressible fluid, N.A.C.A. ARR No. 3G27, 1943. 
36. J. J. Gergen and F. G. Dressel, Mapping by p-regular functions, Duke Math. J. vol. 18 no. 1 (1951) pp. 185-210.

37. — Mapping for elliptic equations, Trans. Amer. Math. Soc. vol. 77 (1954) pp. $151-178$.

38. — Uniqueness for p-regular mapping, Duke Math. J. vol. 19 (1952) pp. 435-444.

39. P. Hartman and A. Wintner, On the third fundamental form of a surface, Amer. J. Math. vol. 75 (1953) pp. 298-334.

40. - On the local behavior of solutions of non-parabolic partial differential equations, ibid. (1953) pp. 449-476.

41. - Umbilical points and W-surfaces, Amer. J. Math. vol. 76 (1954) pp. 502-508.

42. - On the local behavior of solutions of non-parabolic partial differential equations, II; The uniqueness of the Green singularity, Amer. J. Math. vol. 76 (1954) pp. 351-361.

43. E. Heinz, Über die Eindeutigkeit beim Cauchyschen Anfangswertproblem einer elliptischen Differentialgleichung zweiter Ordnung, Nachrichten Goettingen, 1955, No. 1, pp. 1-12.

44. E. Hopf, Elementare Bemerkungen ïber Lösungen partieller Differentialgleichungen zweiter Ordnung vom elliptischen Typus, Preuss. Akad. Wiss. Sitzungsber. vol. 19 (1927) pp. 147-152.

45. F. John, The fundamental solution of linear elliptic differential equations with analytic coefficients, Comm. Pure Appl. Math. vol. 3 (1950) pp. 273-304.

46. S. Kakutani, On the family of pseudo-regular functions, Tôhoku Math. J. vol. 43 (1937) pp. 211-215.

47. W. Kaplan, Topology of level curves of harmonic functions, Trans. Amer. Math. Soc. vol. 63 no. 3 (1948) pp. 514-522.

48. A. Kritszten, Hyperkomplexe und pseudo-analytische Funktionen, Comment. Math. Helv. vol. 26 (1952) pp. 6-35.

49. M. A. Lavrent'ev, A general problem of the theory of quasi-conformal representation of plane regions, Rec. Math. (Mat. Sbornik) N.S. vol. 21 (1947) pp. 285-320.

50. - A fundamental theorem of the theory of quasi-conformal mapping of plane regions, Izvestya Akademii Nauk SSSR. vol. 12 (1948) pp. 513-554.

51. C. Y. Lee, Similarity principle with boundary conditions for pseudo-analylic functions, Duke Math. J. vol. 23 (1956) pp. 157-163.

52. H. Lewy, On differential geometry in the large, I, Trans. Amer. Math. Soc. vol. 43 (1938) pp. 258-270.

53. M. A. Lukomskaya, On one generalization of the class of analytic functions, C.R. (Doklady) Acad. Sci. URSS. vol. 73 (1950) pp. 885-888 (Russian).

54. - On cycles of systems of linear homogeneous differential equations, Rec. Math. (Mat. Sbornik) N.S. vol. 29 (1951) pp. 551-558 (Russian).

55. - Solution of some systems of partial differential equations by means of inclusion in a cycle, Akad. Nauk SSSR. Prikl. Mat. Meh. vol. 17 (1953) pp. 745-747 (Russian).

56. A. Miskis, Uniqueness of the solution of Cauchy's problem, Uspehi Matematičskih Nauk vol. 3 (1948) pp. 3-46 (Russian).

57. C. S. Morawetz, On the non-existence of continuous transonic flows past arbitrary profiles, I. Comm. Pure Appl. Math. vol. 9 (1956) pp. 45-68.

58. - Uniqueness for the analogue of the Neumann problem for mixed equations, Michigan Math. J. (to appear). 
59. C. B. Morrey, On the solutions of quasilinear elliptic partial differential equations, Trans. Amer. Math. Soc. vol. 43 (1938) pp. 126-166.

60. M. Morse, Topological methods in the theory of functions of a complex variable, Annals of Mathematics Studies, no. 15, Princeton University Press, 1947.

61. M. Morse and M. Heins, Topological methods in the theory of functions of a complex variable, Ann. of Math. vol. 46 (1945) pp. 600-624, 625-666; vol. 47 (1946) pp. 233-274.

62. - Deformation classes of meromorphic functions and their extension to interior transformations, Acta. Math. vol. 78-79 (1947) pp. 51-103.

63. M. Morse, and J. A. Jenkins, Multiply-valued harmonic functions and their conjugates on arbitrary Riemann surfaces, Lectures on Functions of a Complex Variable, Michigan University Press, 1955, pp. 123-186.

64. C. Müller, On the behavior of solutions of the differential equation $\Delta U=F(x, U)$ in the neighborhood of a point, Comm. Pure Appl. Math. vol. 7 (1954) pp. 505-515.

65. I. G. Petrovsky, On some problems in the theory of partial differential equations, Uspehi Matematičeskih Nauk vol. 1 (1946) pp. 44-70 (Russian).

66. A. Pfluger, Quasikonforme Abbildungen und logarithmische Kapazität, Annales de l'Institute Fourier, vol. 2 (1950) pp. 69-80.

67. E. Picard, Sur une système des équations aux dérivées partielles, C. R. Acad. Sci. Paris vol. 112 (1891) pp. 685-688.

68. - Sur une gênéralization des equations de la theorie des functions d'une variable complexe, ibid., pp. 1399-1403.

69. G. N. Položii, On p-analytic functions of a complex variable, C. R. (Doklady) Acad. Sci. URSS. vol. 58 (1947) pp. 1275-1278 (Russian).

70. — A generalization of Cauchy's integral formula, Math. Sbornik vol. 24 (1949) pp. 375-384 (Russian).

71. - Singular points and residues of p-analytic functions of a complex variable, C. R. (Doklady) Acad. Sci. URSS. vol. 60 (1949) pp. 769-772 (Russian).

72. - The theorem on preservation of domain for some elliptic systems of differential equations and its applications, Rec. Math. (Mat. Sbornik) N.S. vol. 32 (1953) pp. 485-492 (Russian).

73. M. H. Protter, Generalized spherical harmonics, Trans. Amer. Math. Soc. vol. 63 (1948) pp. 314-341.

74. - The periodicity problem in the theory of pseudoanalytic functions, to appear in Ann. of Math.

75. B. V. Sabat, Cauchy's theorem and formula for linear classes of quasi-conformal mappings, C. R. (Doklady) Acad. Sci. URSS. vol. 69, (1949) pp. 305-308 (Russian).

76. Z. Schapiro, Sur l'existence des représentations quasiconformes, C. R. (Doklady) Acad. Sci. URSS. vol. 30 (1941) pp. 690-692.

77. L. Sobrero, Theorie der ebenen Elastizitaet unter Benutzung eines Systems hyperkomplexer Zahlen, Hamburger Mathematische Einzelschriften, Leipzig, 1934.

78. S. Stollow, Leçons sur les principes topologiques de la theorie des fonctions analytiques, Paris, Gauthier-Villars, 1938.

79. $\mathrm{O}$. Teichmüller, Untersuchungen über konforme und quasikonforme Abbildungen, Deutsche Mathematik vol. 3 (1938) pp. 621-678.

80. I. N. Vekua, New methods for solving elliptic equations, Moscow-Leningrad, 1948 (Russian).

81. - Systems of partial differential equations of first order of elliptic type and boundary value problems with applications to the theory of shells, Rec. Math. (Mat. Sbornik) N.S. vol. 31 (1952) pp. 217-314 (Russian). 
82. - On a property of solutions of the system of generalized Cauchy-Riemann equations, Bull. Acad. Sci. Georgian SSR. vol. 14 (1953) pp. 449-453 (Russian).

83. - General representation of functions of two independent variables admitting derivatives in the sense of S. L. Sobolev, and the problem of primitives, C. R. (Doklady) Acad. Sci. URSS. vol. 89 (1953) pp. 773-775 (Russian).

84. - On some properties of solutions of systems of equations of elliptic type, C. R. (Doklady) Acad. Sci. URSS. vol. 98 (1954) pp. 181-184 (Russian).

85. A. Weinstein, Generalized axially symmetric potential theory, Bull. Amer. Math. Soc. vol. 59 (1953) pp. 20-38.

86. G. T. Whyburn, Introductory topological analsyis, Lectures on Functions of a Complex Variable, Michigan University Press, 1953, pp. 1-14.

REFERENCES ADDED IN PROOF.

87. Nachman Aronszajn, Sur l'unicite du prolongement des solutions des équations aux dérivées partielles elliptiques du second ordre, Comptes rendus vol. 242 (1956) pp. 723-725.

88. Lipman Bers, Formal powers and power series, Comm. Pure Appl. Math. vol. 9 (1956).

89. H. P. Calderon and A. Zygmund, On the existence of certain singular integrals, Acta Math. vol. 88 (1952) pp. 85-139.

90. P. Hartman and A. Wintner, On the local behavior of solutions of non-parabolic partial differential equations. III: Approximations by spherical harmonics, Amer. J. Math. vol. 77 (1955) pp. 453-483.

91. E. M. Landis, On some properties of solutions of elliptic equations, Doklady Acad. Nauk SSSR. vol. 107 (1956) pp. 640-643 (Russian).

\section{NEW YORK UNIVERSITY}

